\title{
POSITIVITY OF THE CM LINE BUNDLE FOR K-STABLE LOG FANOS
}

\author{
QUENTIN POSVA
}

\begin{abstract}
We prove the bigness of the Chow-Mumford line bundle associated to a $\mathbb{Q}$-Gorenstein family of log Fano varieties of maximal variation with uniformly K-stable general geometric fibers. This result generalizes a theorem of Codogni and Patakfalvi to the logarithmic setting.
\end{abstract}

\section{Contents}

1. Introduction 1

2. Preliminaries 4

3. Ampleness lemma 9

4. About the Knudsen-Mumford expansion 10

5. Perturbation of families of K-stable log Fanos 11

6. Proof of the main result $\quad 15$

7. Appendix 26

References 28

\section{INTRODUCTION}

Throughout this article, we work over an algebraically closed field $k$ of characteristic zero.

The notion of K-stability originates from complex analytic geometry. It was first formulated by Tian in [Tia97] to study the existence of Kähler-Einstein metrics on Fano manifolds, and later expressed in algebraic terms by Donaldson in [Don02]. The connection between K-stability and birational geometry, and in particular with the Minimal Model program (MMP), was first noticed several years later: Odaka showed in [Oda13] that K-stable Fano varieties are log terminal, and $\mathrm{Li}$ and $\mathrm{Xu}$ used methods from the MMP to approach questions related to $\mathrm{K}$ stability [LX14]. These were the first steps of a purely algebraic K-stability theory of polarized varieties, with a particular emphasis on the study of K-stability of Fano varieties. Equivalent definitions of K-stability were afterwards formulated in terms of Ding invariant in [Ber16] and of valuation theory [Fuj19a, FO18]. This established a solid ground to study K-stable Fano varieties with methods of birational geometry.

The algebraic geometers' interest for K-stable Fano varieties comes, amongst other reasons, from the possibility of constructing well-behaved moduli spaces. Indeed, after the work of several authors, K-stability appeared to be an adequate global stability condition to obtain compact coarse moduli spaces of Fano varieties. To wit, several compact moduli spaces of del Pezzo K-stable surfaces were constructed [OSS16], as well as the moduli space of smoothable K-polystable Fano varieties [LWX18]. These constructions however rely heavily on techniques from analytic geometry, and it was desirable to find purely algebraic constructions. This program became reality by combining the progress in the algebraic theory of K-stability mentioned above, with the recent breakthroughs in birational geometry (e.g. [BCHM10], [HMX14] and [Bir19]) and in abstract moduli theory ([Alp13] and [AHLH19]). Thanks to several recent works [Jia20, BX19, ABHLX20, BLX19, Xu20, CP21, XZ20, LXZ21], we have now a good understanding of the algebraic moduli functor of K-stable Fano varieties. This article contributes to the study of its compactness properties. 
We shall now explain in more details what is known about the moduli functor of K-stable Fano varieties, and what is our contribution. We refer to Section 2 for the relevant definitions regarding K-stability and birational geometry.

We consider the moduli functor $\mathcal{M}_{n, v, c}^{\mathrm{Kss}}$, where $c \in \mathbb{Q}_{+}$, sending a $k$-scheme $S$ to the set

$$
\mathcal{M}_{n, v, c}^{\text {Kss }}(S)=\left\{\begin{array}{c}
\text { Families }(X, c \Delta) \rightarrow S \text { where }(X, \Delta) \rightarrow S \text { is a } \\
\text { family of log pairs, and for every } t \in T \text { the log } \\
\text { fiber }\left(X_{t}, c \Delta_{t}\right) \text { is a K-semistable log Fano pair } \\
\text { of dimension } n \text { and volume } v .
\end{array}\right\}
$$

It was conjectured that this functor is represented by an Artin stack of finite type over $k$ and admits a projective good moduli space $M_{n, v, c}^{\mathrm{Kps}}$ (in the sense of [Alp13]), whose closed points are in bijection with $n$-dimensional $\mathrm{K}$-polystable $\mathbb{Q}$-Fano varieties of volume $v$. As hinted above, this conjecture is now verified, thanks to the work of several authors:

Theorem 1.0.1 ([Jia20, BX19, ABHLX20, BLX19, Xu20, XZ20, LXZ21]). The moduli functor $\mathcal{M}_{n, v, c}^{K s s}$ is an Artin stack of finite type over $k$ and admits a projective good moduli space $M_{n, v, c}^{K p s}$ whose $k$-points parametrize $K$-polystable $\mathbb{Q}$-Fano varieties of dimension $n$ and volume $v$.

At the time the first version of the present article was written, the above theorem was not yet proved entirely: the missing parts were the properness and projectivity of $M_{n, v, c}^{\mathrm{Kps}}$, which were latter settled through the proof of Finite Generation Conjecture in [LXZ21]. It was also conjectured, and has been verified in full generality in op.cit., that the polarization on the moduli space is given by the so-called Chow-Mumford (CM) line bundle. Our article was part of the effort, together with [CP21] and [XZ20], to show that the CM line bundle is indeed a good candidate.

Before stating our result, let us define the CM line bundle (see also Section 2.4). We consider $f:(X, D) \rightarrow T$ a flat family of $\log$ pairs of relative dimension $n$, such that $X$ and $T$ are projective and normal, and $-\left(K_{X / T}+\Delta\right)$ is $f$-ample. We let

$$
\lambda_{f, D}:=-f_{*}\left(\left(-\left(K_{X / T}+D\right)\right)^{n+1}\right),
$$

where $f_{*}$ is the cycle-pushforward. Then $\lambda_{f, D}$ is a $\mathbb{Q}$-Cartier divisor on $T$, called the CM line bundle of the family $f:(X, D) \rightarrow T$. It has a good functorial behaviour (see Proposition 2.4.1) and therefore defines a $\mathbb{Q}$-line bundle $\lambda$ on $\mathcal{M}_{n, v, c}^{\mathrm{Kss}}$. Better still, it descends to the good moduli space $M_{n, v, c}^{\mathrm{Kps}}$ in the sense that there exists a $\mathbb{Q}$-line bundle $L$ on $M_{n, v, c}^{\mathrm{Kps}}$ whose pullback to $\mathcal{M}_{n, v, c}^{\mathrm{Kss}}$ is $\lambda$ [CP21, Lemma 10.2].

Our main result, which is a step towards the ampleness of $\lambda$, reads as follows:

Theorem 1.0.2. Let $f:(X, D) \rightarrow T$ be a flat morphism of relative dimension $n$ with connected fibers from a normal projective pair to a normal projective variety, such that $-\left(K_{X / T}+D\right)$ is $\mathbb{Q}$-Cartier and $f$-ample. Assume that $D$ does not contain any fibers.

(a) Bigness: If each fiber $\left(X_{t}, D_{t}\right)$ is klt, the general geometric fibers $\left(X_{\bar{t}}, D_{\bar{t}}\right)$ are uniformly $K$-stable, and the variation of $f$ is maximal, then $\lambda_{f, D}$ is big.

(b) AMPLENESS: If all the geometric fibers $\left(X_{\bar{t}}, D_{\bar{t}}\right)$ are uniformly $K$-stable, and the variation of $f$ is maximal, then $\lambda_{f, D}$ is ample.

(Here a general geometric fiber denotes the fiber along a geometric point Spec $\bar{\Omega} \rightarrow U \subseteq X$, where $U \subseteq X$ is a dense open subset and $\bar{\Omega}$ some algebraically closed field.)

The case $D=0$ of Theorem 1.0.2 was proved previously in [CP21, Theorem 1.9]. However that proof does not generalize to the case $D \neq 0$. The difficulty lies in that there exist nonisomorphic log Fano pairs whose underlying varieties are isomorphic, so a family of log Fano pairs $(X, D) \rightarrow T$ can be of maximal variation while the underlying family $X \rightarrow T$ is not. Thus special attention to the geometry of the boundary $D$ is required. Our strategy of proof of Theorem 1.0.2 is explained in Section 1.1: it relies on a perturbative argument on the boundary.

After the first version of this article was put on ArXiv, new positivity results for the CM line bundle were proved in [XZ20]. The authors introduce the notion of reduced uniform K-stability, 
which generalise that of uniform K-stability, and they proved the analogue of Theorem 1.0.2 for families of reduced uniform K-stable log Fano pairs, see [XZ20, §7]. Their strategy to deal with the case $D \neq 0$ was inspired by ours.

Remark 1.0.3. In Theorem 1.0.2, one of our assumptions is that each fiber $\left(X_{t}, D_{t}\right)$ of the family is a klt pair. This hypothesis is natural for applications to moduli space of K-stable Fano varieties, where the families we consider have klt fibers (see Theorem 2.2.3). However it might not be necessary, since in the case $D=0$ we only need the general log fiber to be klt [CP21, Theorem 1.9.a].

\subsection{Overview of the proof}

The proof of the bigness statement is based on the following idea. Let $(X, D) \rightarrow T$ be a $\mathbb{Q}$-Gorenstein family of log Fano pairs of maximal variation with uniformly K-stable general geometric fibers. By [CP21, Theorem 1.8], we know that $\lambda_{f, D}$ is a pseudo-effective divisor. Assume that the components of $D$ are $\mathbb{Q}$-Cartier. Then for a small perturbation $D^{\epsilon}$ of $D$, the perturbed family $\left(X, D^{\epsilon}\right) \rightarrow T$ has the same properties as the original one. Hence the perturbed CM line bundle $\lambda_{f, D^{\epsilon}}$ remains pseudo-effective. By understanding the variation of $\lambda_{f, D}$ into $\lambda_{f, D^{\epsilon}}$, we will deduce that $\lambda_{f, D}$ belongs to the interior of the pseudo-effective cone. If the components of $D$ are not $\mathbb{Q}$-Cartier, we use techniques from the Minimal Model Program (MMP) to run a similar analysis.

1.1.1. Curve base and $\mathbb{Q}$-Cartier coefficients. The variation of $\lambda_{f, D}$ is easy to analyse when the base $T$ is a smooth curve, and all the reduced components $D^{i}$ of $D$ are $\mathbb{Q}$-Cartier. It follows from the definition of the CM line bundle that

$$
-\operatorname{deg} \lambda_{f, D}=\left(-K_{X / T}-D\right)^{n+1}, \quad n+1=\operatorname{dim} X .
$$

Let $D^{\epsilon}=D-\sum_{i} \epsilon_{i} D^{i}$ be a perturbed boundary. As explained above, the divisor $\lambda_{f, D^{\epsilon}}$ is pseudo-effective for small values of $\epsilon$, which means $\operatorname{deg} \lambda_{f, D^{\epsilon}} \geq 0$. We calculate this degree as above:

$$
\begin{aligned}
-\operatorname{deg} \lambda_{f, D^{\epsilon}} & =\left(-K_{X / T}-D+\sum_{i} \epsilon_{i} D^{i}\right)^{n+1} \\
& =\left(-K_{X / T}-D\right)^{n+1}+(n+1) \sum_{i} \epsilon_{i}\left(-K_{X / T}-D\right)^{n} \cdot D^{i}+O\left(\epsilon^{2}\right) \\
& =-\operatorname{deg} \lambda_{f, D}+(n+1) \sum_{i} \epsilon_{i}\left(-K_{X / T}-D\right)^{n} \cdot D^{i}+O\left(\epsilon^{2}\right)
\end{aligned}
$$

Hence for small values of $\epsilon$, the function $\epsilon \mapsto-\operatorname{deg} \lambda_{f, D^{\epsilon}}$ can be approximated by an affine polynomial with linear coefficients $\left(-K_{X / T}-D\right)^{n} \cdot D^{i}$. Assume that these first order derivatives $\left(-K_{X / T}-D\right)^{n} \cdot D^{i}$ are all positive. Then $\operatorname{deg} \lambda_{f, D}$ cannot be too small in comparison to them, for otherwise $\operatorname{deg} \lambda_{f, D^{\epsilon}}<0$ for a small value of $\epsilon$.

We estimate these first order derivatives using the so-called product trick, pioneered in the work of Viehweg [Vie83]. For positive integers $r_{0}, \ldots, r_{N}$, let

$$
D^{\left(r_{\bullet}\right)}=X^{\left(r_{0}\right)} \times_{T}\left(D^{1}\right)^{\left(r_{1}\right)} \times_{T} \cdots \times_{T}\left(D^{N}\right)^{\left(r_{N}\right)}
$$

and let $L$ be the Cartier divisor on $D^{\left(r_{\bullet}\right)}$ given by the sum of the pullbacks of $-K_{X / T}-D$ restricted to the different factors. Then the self-intersection of $L$ depends only on $\left(-K_{X / T}-\right.$ $D)^{n+1},\left(-K_{X / T}-D\right)^{n} \cdot D^{i}$ and $r_{i}$. On the other hand, if $r_{\bullet}$ is suitably chosen, we can infer some positivity of $L$ from the positivity of the sheaf

$$
\operatorname{det}\left(f_{*} \mathcal{O}_{X}\left(-K_{X / T}-D\right) \otimes \bigotimes_{i} f_{*} \mathcal{O}_{D^{i}}\left(-K_{X / T}-D\right)\right)
$$


The positivity of this determinant sheaf is a consequence of the maximal variation assumption via Kollár's ampleness lemma. From the positivity of $L$, we deduce a positive lower bound for the first-order derivatives $\left(-K_{X / T}-D\right)^{n} \cdot D^{i}$.

It is useful in the argument to twist $-K_{X / T}-D$ with a sufficient multiple of $f^{*} \lambda_{f, D}$, since we obtain a nef divisor [CP21, Theorem 1.20]. This replacement has technical significance, but does not affect the strategy.

1.1.2. General case. The CM line bundle behaves well with respect to base-change (Proposition 2.4.1). In particular, it holds that $\lambda_{f, D} \cdot C=\operatorname{deg} \lambda_{f_{C}, D_{C}}$ for a smooth curve $C$ mapping to $T$. Hence if $T$ has higher dimension, we can base-change over a general curve $C$, apply the previous case and obtain $\lambda_{f, D} \cdot C>0$. However, this does not suffice to prove that $\lambda_{f, D}$ is big, as the boundary of the cone of movable curves of $T$ need not be spanned by classes of movable irreducible curves. Nevertheless, this strategy still works if we keep a precise track of the positivity.

(a) First we need to estimate the derivatives $\left(-K_{X_{C} / C}-D_{C}\right)^{n} \cdot D_{C}^{i}$. We can construct $D^{\left(r_{\bullet}\right)}$ and $L$ as before, conclude to some positivity of $L$ and base-change to $C$. However the base-change $D^{\left(r_{\bullet}\right)} \times_{T} C$ might not be flat over a general curve $C$, which creates difficulties. Thus we construct the product from a suitable birational model of $X$ (Notation 6.3.2). Then we use the ampleness lemma and the product trick to estimate the derivatives (Proposition 6.2.1 and Proposition 6.3.5).

(b) We can garantee that these derivatives do not simultaneously go to zero when the class $[C]$ gets closer to the boundary of the movable cone (Lemma 6.3.6). This is done using the theory of Knudsen-Mumford expansion, which is recalled in Section 4.

(c) Once we have a uniform control on the derivatives, we would like to perturb the boundary $D$. However the components $D^{i}$ might not be $\mathbb{Q}$-Cartier. Using the techniques of the MMP, we produce a birational model $W$ of $X$ on which some components become $\mathbb{Q}$ Cartier, and such that the morphism $W \rightarrow T$ has good properties (see Proposition 5.0.4 for the precise statement). Then we are in position to perform the perturbation argument on $W$ (Section 6.4) and conclude.

\subsection{Organization of the paper}

In Section 2 we gather some notations, recall the characterization of K-stability in terms of the $\delta$-invariant and discuss base-change of divisors and the definition of the CM line bundle. The statement of the ampleness lemma is recalled in Section 3, and we gather some facts about the Knudsen-Mumford expansion in Section 4. In Section 5 we show how to perturb a family of pairs so that a part of the boundary becomes $\mathbb{Q}$-Cartier, while the other relevant properties of the family are preserved. Section 6 is devoted to the proof of the main theorem, and the technical results are gathered in the appendix.

\subsection{Acknowledgments}

I would like to thank my advisor Zsolt Patakfalvi for his interest, his support and his help. I am also grateful to Giulio Codogni, Roberto Svaldi and Maciej Zdanowicz for many helpful conversations, and to the anonymous reviewer for numerous corrections and suggestions.

\section{Preliminaries}

\subsection{Notations and conventions}

We fix once and for all an algebraically closed field $k$ of characteristic 0 . Every scheme appearing in this article is a $k$-scheme, and every morphism is a $k$-morphism.

A pair $(X, D)$ is the data of a normal variety $X$ and an effective $\mathbb{Q}$-Weil divisor $D$ such that $K_{X}+D$ is $\mathbb{Q}$-Cartier. We refer to [KM98, §2.3] for the definition of $k l t$ and $l c$ pairs.

A pair $(X, D)$ is Fano if $X$ is projective and $-K_{X}-D$ is ample. A pair $(X, D)$ is weak log Fano if it is a klt projective pair such that $-K_{X}-D$ is big and nef. A pair $(X, D)$ is $\log$ Fano if it is a klt Fano pair. We say that $X$ is $\mathbb{Q}$-Fano if $(X, 0)$ is log Fano. 
If $X$ is a Noetherian variety, an open subset $U \subset X$ is called big if $X \backslash U$ has codimension at least 2 in $X$. More generally, if $f: X \rightarrow T$ is a morphism, then $U$ is relatively big over $T$ if $U_{t} \subset X_{t}$ is big for every $t \in T$.

A birational proper morphism $\pi: Y \rightarrow X$ between projective varieties is called small if the exceptional locus of $\pi$ has codimension at least 2 .

Definition 2.1.1 (General movable curves). Let $X$ be a projective variety. A smooth curve $C \rightarrow X$ is a non-constant morphism (not necessarily an embedding) from a projective smooth curve $C$ to $X$. We say that a smooth curve $C \rightarrow X$ is a general movable curve if it is the normalization of a general curve in a family of curves covering $X$.

Let $Z \subsetneq X$ be a proper closed subset. When fixing a general movable curve, we can always assume that it is not contained in $Z$. By [Laz04b, 11.4.C], a $\mathbb{Q}$-Cartier divisor $D$ on $X$ is big (resp. pseudo-effective) if and only if $D \cdot C>0$ (resp. $D \cdot C \geq 0$ ) for every general movable curve $C \rightarrow X$.

Definition 2.1.2 (Families of log Fano pairs). A $\mathbb{Q}$-Gorenstein family of log Fano pairs $f:(X, D) \rightarrow T$ is the data of a flat projective morphism $f: X \rightarrow T$ between normal projective varieties, and of an effective Weil $\mathbb{Q}$-divisor $D$, such that

(a) the fibers of $f$ are irreducible and normal,

(b) the support of $D$ does not contain any fiber,

(c) $\left(X_{t}, D_{t}\right)$ is klt for each $t \in T$ (the definition of the restricted divisor $D_{t}$ is given in Section 2.3), and

(d) $-K_{X / T}-D$ is an $f$-ample $\mathbb{Q}$-Cartier divisor.

Definition 2.1.3 (Maximal variation). Let $f:(X, D) \rightarrow T$ be a $\mathbb{Q}$-Gorenstein family of $\log$ Fano pairs. Then $f$ has maximal variation if there is a non-empty dense open subset $V \subset T$ such that for every point $t \in V$, the set $\left\{t^{\prime} \in V \mid\left(X_{t}, D_{t}\right) \cong\left(X_{t^{\prime}}, D_{t^{\prime}}\right)\right\}$ is finite.

Notation 2.1.4 (Coefficient parts). Let $X$ be a normal variety and $D$ a Weil $\mathbb{Q}$-divisor on $X$. For $c \in \mathbb{Q}$, the part of coefficient $c$ of $D$ is defined to be

$$
D^{=c}:=\sum_{\operatorname{coeff}_{E} D=c} E
$$

where the sum runs through the set of prime Weil divisors $E$ of $X$. We have $D=\sum_{c \in \mathbb{Q}} c D^{=c}$. For simplicity, if $\left\{c \in \mathbb{Q} \mid D^{=c} \neq 0\right\}=\left\{c_{1}, \ldots, c_{m}\right\}$, we let $D^{i}:=D^{=c_{i}}$ so that $D=\sum_{i=1}^{m} c_{i} D^{i}$. We will also denote by $D^{i}$ the corresponding reduced closed subscheme.

Notation 2.1.5 (Volumes). Let $D$ be a $\mathbb{Q}$-Cartier divisor on a proper scheme $X$. We denote its volume by $\operatorname{Vol}(D)$. We refer to [Laz04a, §2.2.C] for the definition and the properties of the volume.

Notation 2.1.6 (Intersection numbers). Let $D$ be a $\mathbb{Q}$-Cartier divisor on a proper equidimensional scheme $X$ of dimension $n$. We denote by $D^{n}=(D \cdots D)$ its self-intersection. If $D$ is ample, it holds that $\operatorname{Vol}(D)=D^{n}$.

If $X$ is also reduced and $\mathcal{L}$ is a line bundle on $X$, by abuse of notation we denote by $\mathcal{L}$ the associated linear equivalence class of Cartier divisors (see [Liu02, Corollary 1.19]). Then it makes sense to write

$$
\mathcal{L}^{m} \cdot D^{n-m}=(\underbrace{\mathcal{L} \cdots \mathcal{L}}_{m \text { times }} \cdot \underbrace{D \cdots D}_{n-m \text { times }}) .
$$

If $f: C \rightarrow X$ is a morphism from a smooth proper curve, we write

$$
D \cdot C=\frac{1}{r} \operatorname{deg}_{C} f^{*} \mathcal{O}_{X}(r D),
$$

where $r>0$ is such that $r D$ is Cartier.

Notation 2.1.7 ( $m$-fold products). Let $f: X \rightarrow T$ be a morphism of proper schemes. We denote by $X^{(m)}$ the $m$-times fiber product of $X$ with itself over $T$. It comes with projection 
morphisms $p_{i}: X^{(m)} \rightarrow X$ for $i=1, \ldots, m$ and the structural morphism $f^{(m)}: X^{(m)} \rightarrow T$. Given a line bundle $\mathcal{L}$ on $X$, or a Cartier divisor $D$ on $X$, we write

$$
\mathcal{L}^{(m)}:=\bigotimes_{i=1}^{m} p_{i}^{*} \mathcal{L}, \quad D^{(m)}:=\sum_{i=1}^{m} p_{i}^{*} D .
$$

\subsection{K-stability}

In this section, we recall briefly one characterization of the $\delta$-invariant for log Fano pairs, and its relation with K-stability. We refer to [Fuj19b] for the algebraic definition of K-stability in terms of test configurations.

Consider a $n$-dimensional weak $\log$ Fano pair $(X, D)$. Let $E$ be a prime divisor over $X$, and $\pi: Y \rightarrow X$ be a smooth birational model on which $E$ appears. We can write

$$
K_{Y} \equiv_{\text {num }} \pi^{*}\left(K_{X}+D\right)+\sum_{F} a_{X, D}(F) F
$$

where $F$ runs through the prime divisors of $Y$. The log discrepancy of $E$ with respect to $(X, D)$ is

$$
A_{X, D}(E):=a_{X, D}(E)+1
$$

We also define the quantity

$$
S_{X, D}(E):=\frac{1}{\left(-K_{X}-D\right)^{n}} \int_{0}^{+\infty} \operatorname{Vol}\left(\pi^{*}\left(-K_{X}-D\right)-x E\right) d x .
$$

Definition 2.2.1 (Delta invariant). Let $(X, D)$ be a log Fano pair. The $\delta$-invariant of $(X, D)$ is given by

$$
\delta(X, D):=\inf _{E} \frac{A_{X, D}(E)}{S_{X, D}(E)},
$$

where $E$ runs through the prime divisors over $X$.

Remark 2.2.2. The original definition of the delta invariant in [FO18] is formulated in terms of basis-type divisors of the anti-log-canonical linear system. However, the above one is more convenient for our purpose. The equivalence between the two definitions is proved in [BJ20, Theorem 4.4] in the case $D=0$, and [CP21, Theorem 4.6] in the general logarithmic case.

The relation between the delta invariant and K-stability of log Fano pairs is given by the following theorem. In this article, we use this characterization as the definition of uniform Kstability. See [FO18, Theorems 1.1 and 2.1] and [BJ20, Theorem B] for the proof of equivalence.

Theorem 2.2.3. Let $(X, D)$ be a Fano pair.

(a) $(X, D)$ is K-semistable if and only if $(X, D)$ is klt and $\delta(X, D) \geq 1$.

(b) $(X, D)$ is uniformly K-stable if and only if $(X, D)$ is klt and $\delta(X, D)>1$.

The next result will be useful in Section 5 .

Proposition 2.2.4. Let $(X, D)$ be a weak log Fano pair, and $\Gamma$ an effective $\mathbb{Q}$-Cartier divisor supported on $\operatorname{Supp}(D)$. Assume that

$$
\inf _{E} \frac{A_{X, D}(E)}{S_{X, D}(E)}>1
$$

where $E$ runs through the divisors over $X$. Then for all rational $\epsilon>0$ small enough,

$$
\inf _{E} \frac{A_{X, D-\epsilon \Gamma}(E)}{S_{X, D-\epsilon \Gamma}(E)}>1 .
$$

Proof. Replacing $\Gamma$ by a small multiple, we may assume that there is an effective $\mathbb{Q}$-Cartier divisor $\Gamma^{\prime}$ on $X$ such that $\Gamma+\Gamma^{\prime} \in\left|-K_{X}-D\right|_{\mathbb{Q}}$. By assumption, there is an $a>0$ such that $A_{X, D}(E) \geq(1+a) S_{X, D}(E)$ for all divisors $E$. Choose $a^{\prime} \in(0, a)$ and define the function

$$
f(\epsilon):=\left(1+a^{\prime}\right)(1+\epsilon)^{n+1} \frac{\left(-K_{X}-D\right)^{n}}{\left(-K_{X}-D+\epsilon \Gamma\right)^{n}}, \quad \epsilon \in \mathbb{R} .
$$


Since $\lim _{\epsilon \rightarrow 0} f(\epsilon)=1+a^{\prime}$, we can fix $\epsilon_{0}=\epsilon_{0}\left(a^{\prime}\right)>0$ such that for all $\epsilon \in\left(0, \epsilon_{0}\right)$, we have $f(\epsilon)<1+a$. Since we assumed $\operatorname{Supp}(\Gamma) \subset \operatorname{Supp}(D)$, we can also arrange that $D-\epsilon_{0} \Gamma$ is effective.

Now let $E$ be a divisor over $X$, appearing on a birational model $\pi: Y \rightarrow X$. For any $\epsilon>0$ and any $x \in \mathbb{R}_{+}$, observe that

$$
\begin{aligned}
\operatorname{Vol}\left(\pi^{*}\left(-K_{X}-D+\epsilon \Gamma\right)-x E\right) & \leq \operatorname{Vol}\left(\pi^{*}\left(-K_{X}-D+\epsilon\left(\Gamma+\Gamma^{\prime}\right)\right)-x E\right) \\
& =\operatorname{Vol}\left((1+\epsilon) \pi^{*}\left(-K_{X}-D\right)-x E\right) .
\end{aligned}
$$

Integrating over $x$, we obtain

$$
\begin{aligned}
\int_{0}^{\infty} \operatorname{Vol}\left(\pi^{*}\left(-K_{X}-D+\epsilon \Gamma\right)-x E\right) d x & \leq \int_{0}^{\infty} \operatorname{Vol}\left((1+\epsilon) \pi^{*}\left(-K_{X}-D\right)-x E\right) d x \\
& \stackrel{(1+\epsilon) x}{=}(1+\epsilon)^{n+1} \int_{0}^{\infty} \operatorname{Vol}\left(\pi^{*}\left(-K_{X}-D\right)-y E\right) d y .
\end{aligned}
$$

Together with the definition of the functional $S_{X, \bullet}$, the above inequality implies

$$
S_{X, D-\epsilon \Gamma}(E) \leq(1+\epsilon)^{n+1} \frac{\left(-K_{X}-D\right)^{n}}{\left(-K_{X}-D+\epsilon \Gamma\right)^{n}} S_{X, D}(E) .
$$

Now take $\epsilon<\epsilon_{0}$ and assume that $A_{X, D-\epsilon \Gamma}(E) \leq\left(1+a^{\prime}\right) S_{X, D-\epsilon \Gamma}(E)$. Then we have

$$
\begin{aligned}
A_{X, D}(E) \leq A_{X, D}(E)+\epsilon \cdot \operatorname{ord}_{E}(\Gamma)=A_{X, D-\epsilon \Gamma}(E) & \leq\left(1+a^{\prime}\right) S_{X, D-\epsilon \Gamma}(E) \\
& \leq f(\epsilon) S_{X, D}(E) \\
& <(1+a) S_{X, D}(E),
\end{aligned}
$$

and we have obtained a contradiction with the hypothesis. Thus $A_{X, D-\epsilon \Gamma}(E)>\left(1+a^{\prime}\right) S_{X, D-\epsilon \Gamma}$ for all $\epsilon<\epsilon_{0}$. Since $\epsilon_{0}$ does not depend on $E$, the proof is complete.

\subsection{Base-change of divisors}

Notation 2.3.1. In this subsection, we consider a flat morphism $f: X \rightarrow T$ between normal projective varieties, and an effective Weil $\mathbb{Q}$-divisor $D$ on $X$, such that

(a) the fibers of $f$ are connected and normal,

(b) the support of $D$ does not contain any fiber.

Remark 2.3.2. If $(X, D) \rightarrow T$ is as in Notation 2.3.1, then $c D$ is a Mumford divisor in the sense of [Kol19, Definition 1] for $c>0$ divisible enough. Since $T$ is reduced, $c D$ is automatically K-flat over $T$ [Kol19, Definition 2 and paragraph 6]. Thus $\left(X, D=\frac{1}{c} \cdot c D\right) \rightarrow T$ is a K-flat family in the sense of Kollár.

Lemma 2.3.3. In the situation of Notation 2.3.1, each component of D dominates $T$.

Proof. We may assume that $D$ is irreducible. Since $D$ does not contain any component of any fiber, the scheme-theoretic restriction $D \cap X_{t}$ has dimension at most $\operatorname{dim} f-1$ for any $t \in T$. If $D$ does not dominate $T$, then $\operatorname{dim} f(D)<\operatorname{dim} T$. But in this case, for a general point $t \in f(D)$ we have

$$
\operatorname{dim} X-1=\operatorname{dim} D=\operatorname{dim} D \cap X_{t}+\operatorname{dim} f(D)<\operatorname{dim} f-1+\operatorname{dim} T=\operatorname{dim} X-1,
$$

which is a contradiction.

Definition 2.3.4 (Divisorial pullbacks). Let $U \subset X$ be the smooth locus of $f$. By assumption $U$ is relatively big over $T$, thus $f(U)=T$ and $U$ is a big open subset of $X$. By [Kol14, Theorem 4.21], every Weil divisor on $X$ not containing any component of a fiber is Cartier over $U$.

Let $u: S \rightarrow T$ be a morphism from a normal variety $S$. We define the divisorial pullback of $D$ along $u$ as follows. The open set $U_{S}=U \times_{T} S$ is big in $X_{S}=X \times_{S} T$. Since $\left.D\right|_{U}$ is $\mathbb{Q}$-Cartier, it pullbacks to a $\mathbb{Q}$-Cartier divisor $\left.D\right|_{U_{S}}$ on $U_{S}$. We let the divisorial pullback $D_{X_{S}}$ of $D$ along $u$ be the unique Weil $\mathbb{Q}$-divisor extending the $\mathbb{Q}$-Cartier divisor $\left.D\right|_{U_{S}}$.

In particular, if $t \in T$ is a closed point, then $D_{X_{t}}$ is the unique Weil $\mathbb{Q}$-divisor of $X_{t}$ extending the $\mathbb{Q}$-Cartier divisor $\left.D\right|_{U \cap X_{t}}$. For ease of notation, we will mostly write $D_{t}:=D_{X_{t}}$. 
It follows from this definition that there is a $\mathbb{Q}$-linear equivalence

$$
K_{X_{S} / S}+D_{X_{S}} \sim_{\mathbb{Q}} v^{*}\left(K_{X / T}+D\right)
$$

where $v: X_{S} \rightarrow X$ is the induced morphism, see [CP21, §2.4.1].

Lemma 2.3.5. In the situation of Notation 2.3.1, let $S \rightarrow T$ be a morphism from a normal projective variety. If $D$ is Cartier, then the divisorial pullback of $D$ and the pullback of $D$ as Cartier divisor along $\sigma: X_{S} \rightarrow X$ agree.

Proof. If $U$ is the smooth locus of $X \rightarrow T$, then $\sigma^{*} D$ represents $D_{X_{S}}$ on $U_{S}$ by definition. A Cartier divisor on a normal variety is determined in codimension one, and $U_{S}$ is big. Thus $\sigma^{*} D$ represent the Weil divisor $D_{X_{S}}$.

Lemma 2.3.6. In the situation of Notation 2.3.1, there is a dense big open set $U \subseteq T$ over which all the possible unions of components of $D$ (with the reduced structure) are flat.

Proof. Let $E$ be a union of components of $D$ with the reduced structure. By generic flatness, the locus of $T$ over which $E$ is flat, say $U_{E}$, is dense open. Pick a codimension one point $t \in T$, and any $x \in E$ such that $f(x)=t$. The morphism $\mathcal{O}_{T, t} \rightarrow \mathcal{O}_{E, x}$ is flat if and only if the uniformizer $\pi$ of $\mathcal{O}_{T, t}$ is sent to a non-zero-divisor of $\mathcal{O}_{E, x}$. Now if $\pi$ is a zero-divisor in $\mathcal{O}_{E, x}$, then the components of $X_{t}$ passing through $x$ are contained in $E$. But by assumption $X_{t}$ is irreducible and $E$ does not contain any fiber, so this cannot happen. Thus $E \rightarrow T$ is flat at $x$. Since $x$ is arbitrary, we conclude that $t \in U_{E}$. Therefore $U=\bigcap_{E} U_{E}$ is big.

Lemma 2.3.7. In the situation of Lemma 2.3.6, there is a dense open set $V \subseteq U$ such that:

(a) for any $v \in V$ and $c \in \mathbb{Q}$, the divisorial restriction $\left(D^{=c}\right)_{v}$ is equal to the coefficient part $\left(D_{v}\right)=c$.

(b) for any $v \in V$ and $c \in \mathbb{Q}$, the scheme-theoretic fiber $D^{=c} \times k(v)$ is equal to the divisorial restriction $\left(D^{=c}\right)_{v}$ with the reduced structure.

Proof. Base-changing if necessary, we may assume that $U=T$. Given a reduced Weil divisor $E$ not containing any fiber, we claim that the divisorial restriction $E_{t}$ is reduced for a general $t \in T$. In view of Definition 2.3.4, we may assume that $E$ is Cartier. Since the claim is local on $X$, we may assume that $E$ is actually principal, say cut out by $s \in \mathcal{O}(X)$. Then $\mathcal{O}_{X} /(s)$ is reduced and flat over $T$; thus its fiber $\mathcal{O}_{X} /(s) \otimes k(t)$ over a general $t \in T$ is reduced [Gro66, 12.2.1]. This means exactly that the divisor $E_{t}$ is reduced. If $E^{\prime}$ is another reduced divisor not containing any fiber, such that $E$ and $E^{\prime}$ have no common components, by applying the claim to $E+E^{\prime}$ we see that the divisorial restrictions $E_{t}$ and $E_{t}^{\prime}$ have no common component for a general $t \in T$. Let $E$ run through the coefficient parts $D^{=c}$ of $D$ to obtain the first assertion.

If we consider $E$ as a reduced closed subscheme, the scheme-theoretic fiber $E \times k(t)$ has pure codimension one for all $t \in T$ [Har77, III.9.6] and is reduced for a general $t \in T$. Combining this and the first assertion, we obtain the second assertion.

Corollary 2.3.8. In the situation of Lemma 2.3.7, let $C \rightarrow T$ be a smooth curve whose image intersect $V$. Let $Z:=X \times_{T} C$ and $D_{Z}=\sum_{c \in \mathbb{Q}} c\left(D_{Z}\right)^{=c}$ be the divisorial pullback of $D$. Then $\left(D_{Z}\right)^{=c}$ is the divisorial pullback of $D^{=c}$ for all $c \in \mathbb{Q}$.

Proof. We have to check that the divisorial pullbacks of any two distinct coefficient parts $D^{=c}$ and $D^{=c^{\prime}}$, have no component in common. Since these divisorial pullbacks are horizontal over $C$, this can be checked on a general fiber of $Z \rightarrow C$. Since $C$ meets $V$, the result follows from Lemma 2.3.7.

\subsection{The CM line bundle}

Let $f: X \rightarrow T$ be a flat projective morphism of relative dimension $n$ between normal projective varieties, let $D$ be an effective $\mathbb{Q}$-divisor on $X$ such that $-\left(K_{X / T}+D\right)$ is $\mathbb{Q}$-Cartier and $f$-ample. Assume also that the fibers of $f$ are irreducible and normal, and that $\operatorname{Supp}(D)$ does not contain any fiber. Then the Chow-Mumford line bundle of $f:(X, D) \rightarrow T$ is defined to be

$$
\lambda_{f, D}:=-f_{*}\left(\left(-K_{X / T}-D\right)^{n+1}\right)
$$


where $f_{*}$ denotes the pushforward of cycles. By [CP21, Proposition 3.7], $\lambda_{f, D}$ is a $\mathbb{Q}$-Cartier $\mathbb{Q}$-Weil divisor. It is compatible with base-change in the following sense:

Proposition 2.4.1. In the above situation, let $\tau: S \rightarrow T$ be a morphism from a normal variety $S$. Let $f_{S}: X_{S} \rightarrow S$ be the induced morphism and $D_{S}$ be the divisorial pullback in the sense of Section 2.3. Then $\tau^{*} \lambda_{f, D}=\lambda_{f_{S}, D_{S}}$.

We refer to $[\mathrm{CP} 21, \S 3]$ for the proof and more background.

\section{Ampleness Lemma}

The next theorem, which is an elaboration of [Kol90, 3.9], will be useful to establish positivity properties of line bundles.

Let us fix our notations for the Grassmannians and the general linear groups over $k$. Given integers $w \geq q$, we let $\operatorname{Gr}_{k}(w, q)$ be the Grassmannian of $q$-dimensional quotients of a $w$ dimensional $k$-vector space. Given an integer $n$, we let $\mathrm{GL}_{k}(n)$ be the general linear group over $k$ of degree $n$.

Theorem 3.0.1. Let $U$ be a normal variety that can be embedded as a big open subset of a projective variety. Let $W, Q_{1}, \ldots, Q_{s}$ be vector bundles on $U$ of respective ranks $w, q_{1}, \ldots, q_{s}$. Assume that there exist morphisms $\phi_{i}: W \rightarrow Q_{i}$ for $i=1, \ldots, s$ which are generically surjective. Assume also that the classifying map

$$
U(k) \rightarrow\left(\prod_{i=1}^{s} \operatorname{Gr}_{k}\left(w, q_{i}\right)\right) / \mathrm{GL}_{k}(w)
$$

is finite-to-one on a dense subset of $U$. Then for any ample Cartier divisor $B$ on $U$, there exists a positive integer $m>0$ and a non-zero morphism

$$
\operatorname{Sym}^{m q}\left(\bigoplus_{i=1}^{s^{2} w} W\right) \longrightarrow \mathcal{O}_{U}(-B) \otimes\left(\bigotimes_{i=1}^{s} \operatorname{det} Q_{i}\right)^{\otimes m}
$$

where $q=\sum_{i=1}^{s} q_{i}$.

Proof. The proof is contained in [KP17, §5]. More precisely, let $Q_{i}^{\prime}$ be the image of $\phi_{i}: W \rightarrow Q_{i}$, with corestricted morphisms $\phi_{i}^{\prime}: W \rightarrow Q_{i}^{\prime}$. There is a big open subset $U^{\prime} \subset U$ over which $Q_{i}^{\prime}$ is locally free of rank $q_{i}$, for each $i$. Since the statement is about the existence of a non-zero map between two locally free sheaves, by reflexivity we may replace $U$ by $U^{\prime}$ and assume that all $Q_{i}^{\prime}$ are locally free. Now let $W^{\prime}=\oplus_{i=1}^{s} W, Q^{\prime}=\oplus_{i=1}^{s} Q_{i}^{\prime}$ and $\phi^{\prime}=\oplus_{i=1}^{s} \phi_{i}^{\prime}$. As explained in [KP17, Lemma 5.6], $\phi^{\prime}$ is surjective over $U$, and there is a dense open set of $U$ where the classifying map corresponding to $\phi^{\prime}$ has finite fibers. Now we follow the proof of [KP17, Theorem 5.5] applied to $\phi^{\prime}: W^{\prime} \rightarrow Q^{\prime}$. In this proof, the assumption of weak positivity is only used in the last three lines of proof; in particular, the equation (5.5.5) and the subsequent displayed isomorphisms hold without this assumption. Thus they apply to our setting: for some $m>0$, there exists a non-zero morphism

$$
\operatorname{Sym}^{m \cdot r k}\left(Q^{\prime}\right)\left(\bigoplus_{i=1}^{\operatorname{rk}\left(W^{\prime}\right)} W^{\prime}\right) \longrightarrow \mathcal{O}_{U}(-B) \otimes\left(\operatorname{det} Q^{\prime}\right)^{\otimes m}
$$

Moreover, it follows from Lemma 3.0.2 below that we have an inclusion

$$
\mathcal{O}_{U}(-B) \otimes\left(\operatorname{det} Q^{\prime}\right)^{\otimes m} \hookrightarrow \mathcal{O}_{U}(-B) \otimes\left(\bigotimes_{i=1}^{s} \operatorname{det} Q_{i}\right)^{\otimes m} .
$$

The result follows by composing the two morphisms.

Lemma 3.0.2. Let $U$ be a normal variety and $\alpha: Q^{\prime} \hookrightarrow Q$ an inclusion of locally free sheaves. Assume that $\alpha$ is generically surjective. Then $\operatorname{det}\left(Q^{\prime}\right) \hookrightarrow \operatorname{det}(Q)$. 
Proof. Let $\mathcal{F}$ be the cokernel of $\alpha$. Then $\mathcal{F}$ is torsion, and the determinant $\operatorname{sheaf} \operatorname{det}(\mathcal{F})=$ $\operatorname{det}(Q) \otimes \operatorname{det}\left(Q^{\prime}\right)^{-1}$ is of the form $\mathcal{O}_{U}(E)$ for an effective divisor $E$. It follows that $\operatorname{det}\left(Q^{\prime}\right) \cong$ $\mathcal{O}_{U}(-E) \otimes \operatorname{det}(Q)$ embeds into $\mathcal{O}_{U} \otimes \operatorname{det}(Q) \cong \operatorname{det}(Q)$.

\section{About the KnUdsen-Mumford expansion}

In this section, we give an alternative description of the CM line bundle, that will be useful to study its positivity properties.

To begin with, we recall a special case of [KM76, Theorem 4]. Let $f: X \rightarrow T$ be a projective morphism between Noetherian schemes of relative dimension $n$. We do not require that $f$ is flat. Let $A$ be an $f$-very ample Cartier divisor. Then there exist $\mathcal{M}_{i} \in \operatorname{Pic}(T)$ such that for every $q \gg 0$, there is an isomorphism

$$
\operatorname{det} f_{*} \mathcal{O}_{X}(q A) \cong \operatorname{det} R f_{*} \mathcal{O}_{X}(q A) \cong \bigotimes_{i=0}^{n+1} \mathcal{M}_{i}^{\otimes\left(\begin{array}{c}
q \\
i
\end{array}\right)}
$$

We call this expression the Knudsen-Mumford expansion of $\mathcal{O}_{X}(A)$, and refer to the $\mathcal{M}_{i}$ as the coefficients of the expansion. This isomorphism is moreover functorial: if $S \rightarrow T$ is a morphism from a Noetherian scheme and $A_{S}$ the pullback of $A$ to $X \times_{T} S$, then it holds that

$$
\left.\operatorname{det}\left(f_{S}\right)_{*} \mathcal{O}_{X_{S}}\left(q A_{S}\right) \cong \bigotimes_{i=0}^{n+1}\left(\mathcal{M}_{i}\right)_{S}^{\otimes(} \begin{array}{l}
q \\
i
\end{array}\right), \quad q \gg 0 .
$$

Now consider the particular case where $f:(X, D) \rightarrow T$ is a $\mathbb{Q}$-Gorenstein family of log Fano pairs. Let $s$ be such that $s\left(-K_{X / T}-D\right)$ is very ample over $T$. Then one can show that

$$
-s^{n+1} \lambda_{f, D}=\mathcal{M}_{n+1},
$$

where $\mathcal{M}_{n+1}$ is the leading coefficient of the Knudsen-Mumford expansion of $s\left(-K_{X / T}-D\right)$. See [CP21, Proposition 3.7] for a proof.

The following proposition characterizes the numerical class of $\mathcal{M}_{n+1}$ in several situations: when $A$ is nef, or when the morphism $f$ has pleasant properties.

Proposition 4.0.1. Let $f: X \rightarrow T$ be an equidimensional projective morphism of relative dimension $n$ between Noetherian proper schemes (we do not require $f$ to be flat). Let $A$ be an $f$-very ample Cartier divisor on $X$, and $\mathcal{M}_{n+1}$ be the leading coefficient of the KnudsenMumford expansion of $\mathcal{O}_{X}(A)$.

(a) Assume that $A$ is nef. For any smooth curve $C \rightarrow T$, it holds that $\mathcal{M}_{n+1} \cdot C=A_{C}^{n+1}$.

(b) Assume that $A$ is nef and $X$ is generically reduced. Let $X^{\prime} \rightarrow X$ be the normalization morphism, $f^{\prime}: X \rightarrow T$ be the induced morphism and $A^{\prime}$ be the pullback of $A$. Then $\mathcal{M}_{n+1} \cdot C=f_{*}^{\prime}\left(\left(A^{\prime}\right)^{n+1}\right) \cdot C$ for a general movable curve $C \rightarrow T$.

(c) Assume that $T$ is normal and $f$ is flat with normal fibers. Then for any smooth curve $C \rightarrow T$, we have $\mathcal{M}_{n+1} \cdot C=f_{*}\left(A^{n+1}\right) \cdot C=A_{C}^{n+1}$.

Proof. Fix a smooth curve $C \rightarrow T$. In any case, since both $q A$ and $q A_{C}$ are relatively very ample for $q \gg 0$, both sheaves $f_{*} \mathcal{O}_{X}(q A)$ and $\left(f_{C}\right)_{*} \mathcal{O}_{X_{C}}\left(q A_{C}\right)$ are locally free with vanishing $R^{i}, i>0$. It follows from the functoriality of the Knudsen-Mumford expansion that

$$
\operatorname{det}\left[f_{*} \mathcal{O}_{X}(q A)\right] \cdot C=\operatorname{deg} \operatorname{det}\left[\left(f_{C}\right)_{*} \mathcal{O}_{X_{C}}\left(q A_{C}\right)\right], \quad q \gg 0 .
$$

With this set-up:

(a) Assume that $A$ is nef. The left-hand side of equation (4.0.1.b) is given by

$$
\sum_{i=0}^{n+1}\left(\begin{array}{l}
q \\
i
\end{array}\right) \mathcal{M}_{i} \cdot C=\frac{q^{n+1}}{(n+1) !} \mathcal{M}_{n+1} \cdot C+O\left(q^{n}\right)
$$


where $\mathcal{M}_{i}$ are the Knudsen-Mumford coefficients of $\mathcal{O}_{X}(A)$. Now consider the righthand side of the same equation. By Riemann-Roch, for $q$ large enough we have $h^{0}\left(X, \mathcal{O}_{X_{C}}\left(q A_{C}\right)\right)=\operatorname{deg} \operatorname{det}\left[\left(f_{C}\right)_{*} \mathcal{O}_{X_{C}}\left(q A_{C}\right)\right]+\chi\left(C, \mathcal{O}_{C}\right) \cdot \operatorname{rk}\left(f_{C}\right)_{*} \mathcal{O}_{X_{C}}\left(q A_{C}\right)$.

Since $A_{C}$ is nef, we have

$$
h^{0}\left(X, \mathcal{O}_{X_{C}}\left(q A_{C}\right)\right)=\frac{q^{n+1}}{(n+1) !} A_{C}^{n+1}+O\left(q^{n}\right)
$$

by [Kol96, VI.2.15]. Since the fibers of $f$ are $n$-dimensional, the function

$$
q \mapsto \operatorname{rk}\left(f_{C}\right)_{*} \mathcal{O}_{X_{C}}\left(q A_{C}\right)
$$

is a polynomial in $q$ of degree at most $n$. Hence

$$
\operatorname{deg} \operatorname{det}\left[\left(f_{C}\right)_{*} \mathcal{O}_{X_{C}}\left(q A_{C}\right)\right]=\frac{q^{n+1}}{(n+1) !} A_{C}^{n+1}+O\left(q^{n}\right) .
$$

It follows by comparing the leading coefficients in (4.0.1.b) that $A_{C}^{n+1}=\mathcal{M}_{n+1} \cdot C$.

(b) Assume that $A$ is nef and $X$ generically reduced. The normalization morphism $X^{\prime} \rightarrow X$ is finite, so $A^{\prime}$ is nef and relatively ample over $T$. Say that $s A^{\prime}$ is relatively very ample for some $s>0$, and let $\mathcal{M}_{n+1}^{\prime}$ be the leading coefficient of the Knudsen-Mumford polynomial of $\mathcal{O}_{X^{\prime}}\left(s A^{\prime}\right)$. Since $X$ is generically reduced, the normalization $X^{\prime} \rightarrow X$ is an isomorphism away from a closed subset $Z \subsetneq X$. If $C \rightarrow T$ is a smooth curve which intersects $f(X-Z)$, the pullback morphism $\left(X^{\prime}\right)_{C} \rightarrow X_{C}$ is birational. Using the first assertion, we obtain that

$$
\mathcal{M}_{n+1} \cdot C=A_{C}^{n+1}=\left(A^{\prime}\right)_{C}^{n+1}=s^{-n-1} \mathcal{M}_{n+1}^{\prime} \cdot C .
$$

By [CP21, Lemma A.2] it holds that $\mathcal{M}_{n+1}^{\prime}=f_{*}^{\prime}\left(s A^{\prime}\right)^{n+1}$, so the second assertion follows.

(c) Assume that $T$ is normal and $f$ is flat with normal fibers. Then both $X$ and $X_{C}$ are normal. It follows from [CP21, Lemma A.2] that for $q \gg 0$

$$
\operatorname{det} f_{*} \mathcal{O}_{X}(q A)=\frac{q^{n+1}}{(n+1) !} f_{*}\left(A^{n+1}\right)+O\left(q^{n}\right)
$$

and

$$
\operatorname{det}\left(f_{C}\right)_{*} \mathcal{O}_{X_{C}}\left(q A_{C}\right)=\frac{q^{n+1}}{(n+1) !}\left(f_{C}\right)_{*}\left(A_{C}^{n+1}\right)+O\left(q^{n}\right)
$$

in the Chow groups of $T$ and $C$ respectively. It follows that $\mathcal{M}_{n+1}=f_{*}\left(A^{n+1}\right)$, and by intersecting with $C$ that

$$
\mathcal{M}_{n+1} \cdot C=f_{*}\left(A^{n+1}\right) \cdot C=\operatorname{deg}\left(f_{C}\right)_{*}\left(A_{C}^{n+1}\right)=A_{C}^{n+1}
$$

as claimed.

\section{Perturbation of families of K-stable log fanos}

Consider a $\mathbb{Q}$-Gorenstein family $(X, D) \rightarrow T$ of $\log$ Fano pairs of maximal variation, with uniformly K-stable general fibers. We show in Proposition 5.0.4 below that we can find a model of $(X, D)$ with the same properties over $T$, and on which some components of the boundary $D$ become $\mathbb{Q}$-Cartier.

We need a few preliminary lemmas. For the first one, we use the terminology of [BCHM10].

Lemma 5.0.1. Let $f: X \rightarrow T$ be a projective morphism between quasi-projective normal varieties. Let $D$ be an effective $\mathbb{Q}$-Cartier $\mathbb{Q}$-divisor on $X$ such that $(X, D)$ is (klt) weak log Fano over $T$. Assume that $D_{1}, \ldots, D_{m}$ are effective $\mathbb{Q}$-Cartier $\mathbb{Q}$-divisors on $X$ with supports contained in the support of $D$. Then there exists a full-dimensional closed polytope $P \subset\left(\mathbb{R}_{\geq 0}\right)^{m}$ with the following properties: 
(a) $P$ contains the origin, and its interior $\operatorname{int}(P)$ is contained in $\left(\mathbb{R}_{>0}\right)^{m}$; and

(b) for every rational vector $\left(\epsilon_{1}, \ldots, \epsilon_{m}\right) \in \operatorname{int}(P)$, the log canonical models of $\left(-K_{X}-D+\right.$ $\left.\sum_{i} \epsilon_{i} D_{i}\right)$ over $T$ have isomorphic underlying varieties.

Proof. Fix a general very ample divisor $A$ on $X$, which has no component in common with $D$. Since $X$ is of Fano type over $T$, there is a $\mathbb{Q}$-boundary $\Delta$ such that $(X, \Delta)$ is klt, $\Delta$ is big over $T$ and $a\left(-K_{X}-D-A\right) \sim_{\mathbb{Q}, T} K_{X}+\Delta$ for some small rational $a>0$. Replacing $A$ by a general member of its linear system, we may assume that $(X, \Delta+a A)$ is also klt [KM98, 5.17]. We have $K_{X}+\Delta+a A+\sum_{i} \epsilon_{i} D_{i} \sim_{\mathbb{Q}, T} a\left(-K_{X}-D\right)+\sum_{i} \epsilon_{i} D_{i}$. So a log canonical model of $\left(X, \Delta+a A+\sum_{i} \epsilon_{i} D_{i}\right)$ over $T$ is also a $\left(-K_{X}-D+\sum_{i} \frac{\epsilon_{i}}{a} D_{i}\right)$-log canonical model of $X$ over $T$. Therefore it is equivalent to prove that: there is a full-dimensional closed polytope $P \subset\left(\mathbb{R}_{>0}\right)^{m}$ containing the origin, such that for all $\left(\epsilon_{1}, \ldots, \epsilon_{m}\right) \in \operatorname{int}(P)$, the pairs $\left(X, \Delta+a A+\sum_{i} \epsilon_{i} D_{i}\right)$ have a $\log$ canonical model over $T$ with isomorphic underlying varieties.

Let us write $A^{\prime}:=a A$ and define the affine cone $V:=\Delta+\sum_{i} \mathbb{R}_{+} D_{i}$ in Weil $(X)_{\mathbb{R}}$. Since $\left(X, \Delta+A^{\prime}\right)$ is klt, there is an open Euclidean neighborhood $\mathcal{U}$ of $\Delta \in V$ such that for all $\Gamma \in \mathcal{U}$, the pair $\left(X, \Gamma+A^{\prime}\right)$ is klt. Also, since $K_{X}+\Delta+A^{\prime} \sim_{\mathbb{Q}, T} a\left(-K_{X}-D\right)$ is big over $T$, we may shrink $\mathcal{U}$ so that $K_{X}+\Gamma+A^{\prime}$ is big over $T$ for all $\Gamma \in \mathcal{U}$. With the notations of [BCHM10, 1.1.4], this implies that $\mathcal{U} \subset \mathcal{E}_{A^{\prime}, f}(V)$.

It follows from [BCHM10, Corollary 1.1.5 and Theorem E] that there are finitely birational contractions $\psi_{i}: X \rightarrow Z_{i}$ over $T, i=1, \ldots, n$, and a decomposition

$$
\mathcal{E}_{A^{\prime}, f}(V)=\bigcup_{i=1}^{n} \mathcal{W}_{i}
$$

where each $\mathcal{W}_{i}=\mathcal{W}_{\psi_{i}, A^{\prime}, f}(V)$ is a rational polytope, such that for each $\Gamma \in \mathcal{W}_{i}$, the underlying variety of a weak log canonical model of $\left(X, \Gamma+A^{\prime}\right)$ over $T$ is isomorphic to $Z_{i}$.

By [BCHM10, Theorem 1.2], for every $\Gamma \in \mathcal{U}$ the pair $\left(X, \Gamma+A^{\prime}\right)$ has a log canonical model over $T$. Relative $\log$ canonical models are in particular relative weak log canonical models. So we obtain that for any $\Gamma \in \mathcal{U} \cap \mathcal{W}_{i}$, the underlying variety of a log canonical model of $\left(X, \Gamma+A^{\prime}\right)$ is isomorphic to $Z_{i}$.

Since $\mathcal{E}_{A^{\prime}, f}(V)$ contains the open neighborhood of the origin of $V$, there must be a polytope $\mathcal{W}_{i}$ which is of full dimension in $V$ and whose closure contains the origin of $V$. Thus we may find a closed full-dimensional polytope $P \subset\left(\mathbb{R}_{\geq 0}\right)^{m}$ containing the origin, with non-empty interior $\operatorname{int}(P) \subset\left(\mathbb{R}_{>0}\right)^{m}$ such that

$$
\left(\bigcup_{\left(\epsilon_{1}, \ldots, \epsilon_{m}\right) \in \operatorname{int}(P)} \Delta+\sum_{i} \epsilon_{i} D_{i}\right) \subset \mathcal{U} \cap \mathcal{W}_{i} \text { for some } i \text {. }
$$

This finishes the proof.

Lemma 5.0.2. Let $(X, D) \rightarrow T$ be a $\mathbb{Q}$-Gorenstein family $(X, D) \rightarrow T$ of log Fano pairs of maximal variation. Write $D=\sum_{i} c_{i} D^{i}$ as in Notation 2.1.4. Then there is a rational number $r>0$ such that for all $i$ such that $D^{i}$ is $\mathbb{Q}$-Cartier, and for all rational $\epsilon \in(-r ; r)$, the family $\left(X, D+\epsilon D^{i}\right) \rightarrow T$ has maximal variation.

Proof. Take $r=\min _{i \neq j}\left\{\frac{1}{2}\left|c_{i}-c_{j}\right|\right\}$.

Lemma 5.0.3. Let $f:(X, D) \rightarrow T$ be a flat equidimensional proper morphism from a normal pair to a smooth variety. Assume that every fiber $\left(X_{t}, D_{t}\right)$ is klt. Then:

(a) $(X, D)$ is klt, and

(b) for any closed point $t \in T$, if $H_{1}, \ldots, H_{d}(d=\operatorname{dim} T)$ are general Cartier divisors in a base-point free linear system such that in a neighbourhood of $t$ we have $\bigcap_{i} H_{i}=\{t\}$, then $\left(X, D+\sum_{i} f^{*} H_{i}\right)$ is dlt in a neighborhood of $X_{t}$.

Proof. Let $t \in T$ be a closed point, and let $H_{1}, \ldots, H_{d}(d=\operatorname{dim} T)$ be general Cartier divisors on $T$ such that $\bigcap_{i} H_{i}=\{t\}$.

To begin with, we prove that $(X, D)$ is klt. Indeed, we can choose the $H_{1}, \ldots, H_{d-1}$ in a general linear system, so the iterated hyperplane sections $X^{m}:=\bigcap_{i=1}^{m} f^{*} H_{i}$ are normal varieties 
for $m \leq d-1$ [Sei50]. By inversion of adjunction, since $\left(X_{t}, D_{t}\right)$ is assumed to be klt, we obtain that $\left(X^{d-1},\left.D\right|_{X^{d-1}}+f^{*} H_{d}\right)$ is plt along $X_{t}$ [KM98, Theorem 5.50]. Hence $\left(X^{d-1},\left.D\right|_{X^{d-1}}\right)$ is klt along $X_{t}$. We repeat this argument to obtain that $(X, D)$ is klt along $X_{t}$. The choice of $t$ was arbitrary, so we conclude that $(X, D)$ is klt.

Now we think of $t \in T$ as a fixed point and claim that if the $H_{i}$ are suitably chosen, then the pair $\left(X, D+\sum_{i=1}^{d} f^{*} H_{i}\right)$ is dlt in a neighborhood of $X_{t}$. Indeed, we can choose the $H_{m}$ inductively with the property that

for each $I \subseteq\{1, \ldots, m\}$, the intersection $X^{I}:=\bigcap_{i \in I} f^{*} H_{i}$ is irreducible and normal.

Each of these conditions is satisfied for a general $H_{m}$ passing through $t$, except for the condition on $H_{d}$ that $\bigcap_{i=1}^{d} f^{*} H_{i}=X_{t}$ is irreducible and normal. But this is satisfied for any choice of $H_{d}$, since $X_{t}$ is assumed to be irreducible and normal.

It follows from the choices of these $H_{i}$ that $\left(X, D+\sum_{i=1}^{d} f^{*} H_{i}\right)$ is snc at every generic point of the $X^{I}$. If $E$ is an exceptional divisor over $X$ whose center $c_{X}(E)$ belongs to $X_{t}$ but does not belong to the snc locus of $\left(X, D+\sum_{i} f^{*} H_{i}\right)$, then $c_{X}(E)$ defines a point of codimension $\geq 1$ in $X_{t}$, and by adjunction we obtain that $\left(X_{t}, D_{t}\right)$ is not klt, which is a contradiction.

Proposition 5.0.4. Let $f:(X, D) \rightarrow T$ be a $\mathbb{Q}$-Gorenstein family of log Fano pairs of maximal variation with uniformly $K$-stable general geometric fiber. Assume that $T$ is smooth. Then there exists a positive number $r_{X, D}>0$ with the following property. For every coefficient part $\Gamma:=D^{i}$ of $D$ (as in Notation 2.1.4), there exists a small proper birational morphism $\nu: W \rightarrow X$ such that:

(a) the strict transform $\Gamma_{W}$ of $\Gamma$ is $\mathbb{Q}$-Cartier, and

(b) for any rational $0<\epsilon<r_{X, D}$, the family $\left(W, D_{W}-\epsilon \Gamma_{W}\right) \rightarrow T$ is a $\mathbb{Q}$-Gorenstein family of log Fano pairs of maximal variation with uniformly $K$-stable general geometric fibers. (Here $D_{W}$ denotes the strict transform of D.)

Proof. Since there are finitely many coefficient part of $D$, we only need to prove the result for a fixed $\Gamma$. First we construct $\nu: W \rightarrow X$.

○ The pair $(X, D)$ is klt by Lemma 5.0 .3 , so by [Kol13, Corollary 1.37 ] there is a small proper birational morphism $\mu: Y \rightarrow X$ where $Y$ is a $\mathbb{Q}$-factorial projective variety. Denote by $D_{Y}$ the strict transform of $D$, and $\Gamma_{Y}$ the strict transform of $\Gamma$. We have

$$
\mu^{*}\left(K_{X}+D\right) \sim_{\mathbb{Q}} K_{Y}+D_{Y} .
$$

○ For $\epsilon>0$, run a $\left(-K_{Y}-D_{Y}+\epsilon \Gamma_{Y}\right)$-MMP over $X$ to obtain a relative log canonical model. By Lemma 5.0.1, this model $W$ is the same for all $0<\epsilon \ll 1$. Denote by $p: Y \rightarrow W$ the induced morphism and $D_{W}:=p_{*} D_{Y}, \Gamma_{W}:=p_{*} \Gamma_{Y}$.

Our construction is pictured by the following diagram:

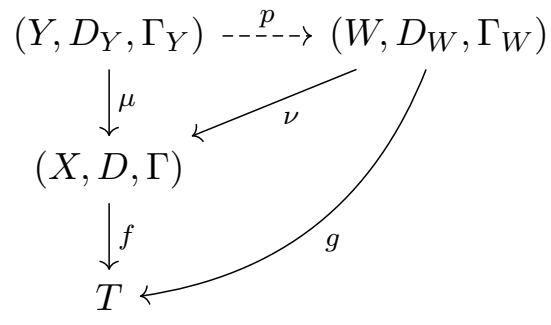

We must show that for smal rationall $\epsilon>0$, the morphism $\left(W, D_{W}-\epsilon \Gamma_{W}\right) \rightarrow T$ is flat between normal projective varieties, of maximal variation, with (klt) $\log$ Fano fibers and uniformly $\mathrm{K}$-stable general geometric fibers, and that $\Gamma_{W}$ is $\mathbb{Q}$-Cartier. First we establish the global properties of $W$ and $\Gamma_{W}$.

- The morphism $\nu: W \rightarrow X$ is small. Indeed, $\mu$ is small and $p$ extracts no divisors. Notice that $D_{W}$ is equal to the strict transform of $D$.

- Since $W$ is the end product of an MMP, it is normal. Moreover, since $\nu$ is small and $(X, D)$ is klt by Lemma 5.0.3, $\left(W, D_{W}\right)$ is klt and hence $W$ is Cohen-Macaulay. 
- The $\mathbb{Q}$-divisor $\Gamma_{W}$ is $\mathbb{Q}$-Cartier. Indeed, $p_{*}\left(-K_{Y}-D_{Y}+\epsilon \Gamma_{Y}\right)=-K_{W}-D_{W}+\epsilon \Gamma_{W}$ is $\mathbb{Q}$ Cartier by construction. It holds that $\nu^{*}\left(-K_{X}-D\right)=-K_{W}-D_{W}$, and so $-K_{W}-D_{W}$ is also $\mathbb{Q}$-Cartier. Therefore $\Gamma_{W}$ is $\mathbb{Q}$-Cartier.

$\circ$ We have

$$
\epsilon \Gamma_{Y} \equiv_{X}-K_{Y}-\left(D_{Y}-\epsilon \Gamma_{Y}\right)
$$

and thus

$$
\epsilon \Gamma_{W} \equiv_{X}-K_{W}-\left(D_{W}-\epsilon \Gamma_{W}\right) \text { is ample over } X .
$$

Hence $\Gamma_{W}$ is a $\mathbb{Q}$-Cartier $\mathbb{Q}$-divisor which is ample over $X$. Furthermore,

$$
\begin{aligned}
-K_{W}-\left(D_{W}-\epsilon \Gamma_{W}\right) & =p_{*}\left(-K_{Y}-\left(D_{Y}-\epsilon \Gamma_{Y}\right)\right) \\
& =p_{*}\left(p^{*} \nu^{*}\left(-K_{X}-D\right)+\epsilon \Gamma_{Y}\right) \\
& =\nu^{*}\left(-K_{X}-D\right)+\epsilon \Gamma_{W} .
\end{aligned}
$$

Now by [Pat15, Lemma 2.4],

$$
-K_{W}+g^{*} K_{T}=-K_{W / T}, \quad-K_{X}+f^{*} K_{T}=-K_{X / T} .
$$

Hence

$$
-K_{W / T}-\left(D_{W}-\epsilon \Gamma_{W}\right)=\nu^{*}\left(-K_{X / T}-D\right)+\epsilon \Gamma_{W}
$$

and therefore $-K_{W / T}-D_{W}+\epsilon \Gamma_{W}$ is ample over $T$ for $0<\epsilon \ll 1$.

To study the properties of a fiber $W_{t}$, we may shrink $T$ and work in a neighborhood of $W_{t}$. Let $H_{1}, \ldots, H_{d}$ be general Cartier divisors such that $\bigcap_{i} H_{i}=\{t\}$.

- The fibers of $\nu$ are connected by construction. Since the fibers of $f$ are irreducible, we deduce that $g$ has connected fibers.

- Since $\nu$ is small, it gives a crepant morphism from $\left(W, D_{W}+\sum_{i=1}^{d} g^{*} H_{i}\right)$ to $(X, D+$ $\left.\sum_{i=1}^{d} f^{*} H_{i}\right)$. Moreover $\nu$ is an isomorphism above the snc locus of $\left(X, D+\sum_{i} f^{*} H_{i}\right)$ [Deb01, 1.40]. By Lemma 5.0.3 the pair $\left(X, D+\sum_{i} f^{*} H_{i}\right)$ is dlt. Thus $\left(W, D_{W}+\right.$ $\left.\sum_{i=1}^{d} g^{*} H_{i}\right)$ is lc, and every lc center is contained in the locus where $\nu$ is an isomorphism. It follows that $\left(W, D_{W}+\sum_{i=1}^{d} g^{*} H_{i}\right)$ is also dlt.

- From $\left[\right.$ Kol13, 4.16] we deduce that every irreducible component of $W_{t}=\bigcap_{i=1}^{d} g^{*} H_{i}$ is normal of codimension $d$, and an lc center of $\left(W, D_{W}+\sum_{i=1}^{d} g^{*} H_{i}\right)$. Assume that $W_{t}$ has at least two different components; by connectedness of the fibers of $W \rightarrow T$, the two components must intersect, and the intersection is a union of lc centers of $\left(W, D_{W}+\sum_{i=1}^{d} g^{*} H_{i}\right)[$ Kol13, 4.20.2]. Since $(X, D)$ is klt by Lemma 5.0.3, we have $\lfloor D\rfloor=0$ and thus $\left\lfloor D_{W}\right\rfloor=0$. So by [Kol13, 4.16.1] the components of $W_{t}$ are minimal lc centers, and we have reached a contradiction. Hence $W_{t}$ is irreducible and normal of codimension $d$.

- Since $W$ is Cohen-Macaulay, $T$ smooth and the fibers $W_{t}$ equidimensional, the morphism $g: W \rightarrow T$ is flat [Mat89, Theorem 23.1].

- Assume that some fiber $W_{t}$ is contained in the support of $D_{W}$. Since $W_{t}$ dominates $X_{t}$ and $D_{W}$ dominates $D$, we obtain that $X_{t}$ is contained in the support of $D$, which is impossible. Thus $\operatorname{Supp}\left(D_{W}\right)$ contains no fibers of $W \rightarrow T$.

Finally we study the pairs $\left(W_{t},\left(D_{W}-\epsilon \Gamma_{W}\right)_{t}\right)$.

○ We know that $\left(W, D_{W}+\sum_{i} g^{*} H_{i}\right)$ is dlt with reduced boundary $\sum_{i} g^{*} H_{i}$. Combining adjunction and $[\mathrm{Kol13}, 4.16]$, we obtain that the pair $\left(W_{t},\left(D_{W}\right)_{t}\right)$ is klt. Hence $\left(W_{t},\left(D_{W}-\epsilon \Gamma_{W}\right)_{t}\right)$ is klt for every $0<\epsilon<\operatorname{coeff}_{\Gamma_{W}} D_{W}[\mathrm{KM} 98,2.27]$.

- Since $\mu$ is small, for a general $t \in T$ the morphism $W_{t} \rightarrow X_{t}$ is small and $\left(D_{W}\right)_{t}$ is the strict transform of $D_{t}$. Fix one such $t$ for which $\left(X_{t}, D_{t}\right)$ is uniformly K-stable. Then for every prime divisor $E$ over $W_{t}$, we have

$$
A_{W_{t},\left(D_{W}\right)_{t}}(E)=A_{X_{t}, D_{t}}(E), \quad S_{W_{t},\left(D_{W}\right)_{t}}(E)=S_{X_{t}, D_{t}}(E),
$$


so by Theorem 2.2.3 we have

$$
\inf _{E} \frac{A_{W_{t},\left(D_{W}\right)_{t}}(E)}{S_{W_{t},\left(D_{W}\right)_{t}}(E)}=\inf _{E} \frac{A_{X_{t}, D_{t}}(E)}{S_{X_{t}, D_{t}}(E)}=\delta\left(X_{t}, D_{t}\right)>1 .
$$

Moreover $\left(W_{t},\left(D_{W}\right)_{t}\right)$ is a weak log Fano pair. Thus we may apply Proposition 2.2.4 to obtain that

$$
\delta\left(W_{t},\left(D_{W}\right)_{t}-\epsilon \Gamma_{t}\right)=\inf _{E} \frac{A_{W_{t},\left(D_{W}\right)_{t}-\epsilon \Gamma_{t}}(E)}{S_{W_{t},\left(D_{W}\right)_{t}-\epsilon \Gamma_{t}}(E)}>1
$$

for all $\epsilon>0$ small enough depending on $t$. Since $\left(W_{t},\left(D_{W}\right)_{t}-\epsilon \Gamma_{t}\right)$ is a log Fano pair, we conclude by Theorem 2.2.3 that $\left(W_{t},\left(D_{W}\right)_{t}-\epsilon \Gamma_{t}\right)$ is uniformly K-stable for all $\epsilon=\epsilon(t)>0$ small enough.

- By openness of the uniform K-stable locus [BL18, Theorem 6.8], we conclude that: for all rational $0<\epsilon \ll 1$, the general fiber of the family $\left(W, D_{W}-\epsilon \Gamma_{W}\right) \rightarrow T$ is uniformly K-stable.

- For a general $t \in T$, the pair $\left(X_{t}, D_{t}\right)$ is a log canonical model of $\left(W_{t},\left(D_{W}\right)_{t}\right)$. Thus $\left(W_{t},\left(D_{W}\right)_{t}\right) \cong\left(W_{u},\left(D_{W}\right)_{u}\right)$ implies that $\left(X_{t}, D_{t}\right) \cong\left(X_{u}, D_{u}\right)$. Moreover by Lemma 5.0.2, for $\epsilon$ small enough, $\left(W_{t},\left(D_{W}-\epsilon \Gamma_{W}\right)_{t}\right) \cong\left(W_{u},\left(D_{W}-\epsilon \Gamma_{W}\right)_{u}\right)$ if and only if $\left(W_{t},\left(D_{W}\right)_{t}\right) \cong$ $\left(W_{u},\left(D_{W}\right)_{u}\right)$. Therefore $\left(W, D_{W}-\epsilon \Gamma_{W}\right) \rightarrow T$ has maximal variation for $0<\epsilon \ll 1$.

This shows that $\left(W, D_{W}-\epsilon \Gamma_{W}\right) \rightarrow T$ has the required properties for all rational numbers $0<\epsilon \ll 1$.

\section{Proof of the main Result}

This section is devoted to the proof of Theorem 1.0.2, which we divide into several steps. See Section 1.1 for an overview of the strategy. In Section 6.1, we set-up the notational framework of the proof. We use the ampleness lemma in Section 6.2 to obtain the positivity of some relevant sheaf. The estimates of the derivatives using the product trick is obtained in Section 6.3, and the perturbation argument is given in Section 6.4.

\subsection{General notations}

Notation 6.1.1. Let $T$ be a smooth variety and $f:\left(X, D=\sum_{i=1}^{N} c_{i} D^{i}\right) \rightarrow T$ be a $\mathbb{Q}$ Gorenstein family of log Fano pairs of maximal variation with uniformly K-stable general geometric fibers. Here $D^{i}$ is the part of coefficient $c_{i}$, see Notation 2.1.4. We introduce the following additional notations, and shall use them for the rest of this section.

(a) Let $n:=\operatorname{dim} X-\operatorname{dim} T$ and $v:=\left(\left.\left(-K_{X / T}-D\right)\right|_{X_{t}}\right)^{n}$. We write $\delta:=\delta\left(X_{\bar{\eta}}, D_{\bar{\eta}}\right)$ where $\eta$ is the generic point of $T$. Then $\delta$ is the value of $\delta\left(X_{t}, D_{t}\right)$ for a very general point $t \in T$ [CP21, Proposition 4.15].

(b) We let $\lambda_{f, D}:=-f_{*}\left(\left(-K_{X / T}-D\right)^{n+1}\right)$ be the CM line bundle provided by the family $f:(X, D) \rightarrow T$.

(c) The restrictions of the morphism $f$ to the support of the $D^{i}$ (with the reduced structure) are denoted $f_{i}: D^{i} \rightarrow T$. We also write $D^{0}=X$ and $f_{0}=f$. By Lemma 2.3.3, each $f_{i}$ is surjective.

(d) We fix a rational number $\alpha>\max \left\{1, \frac{\delta}{(\delta-1) v(n+1)}\right\}$. Notice that by [CP21, Theorems 1.8 and 1.20], for any $\alpha^{\prime} \geq \alpha$ the $\mathbb{Q}$-Cartier divisor $-K_{X / T}-D+\alpha^{\prime} f^{*} \lambda_{f, D}$ is nef.

Notation 6.1.2. In the situation of Notation 6.1.1, let $\iota: C \rightarrow T$ be a smooth curve. Form the Cartesian square

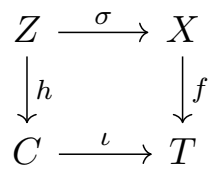

Note that $Z$ is normal because $h$ is flat and its fibers are normal. Let $D_{Z}$ be the divisorial pullback of $D$ (see Definition 2.3.4), with coefficient parts $D_{Z}^{i}$. According to (2.3.4.a) and to 
Proposition 2.4.1, we have

$$
K_{Z / C}+D_{Z} \sim_{\mathbb{Q}} \sigma^{*}\left(K_{X / T}+D\right), \quad \iota^{*} \lambda_{f, D}=\lambda_{h, D_{Z}} .
$$

\subsection{Application of the ampleness lemma}

Proposition 6.2.1. In the situation of Notation 6.1.1, for $q$ divisible enough, the line bundle

$$
\bigotimes_{i \geq 0} \operatorname{det}\left(f_{i}\right)_{*} \mathcal{O}_{D^{i}}\left(q\left(-K_{X / T}-D+2 \alpha f^{*} \lambda_{f, D}\right)\right)
$$

is big on $T$.

The argument of the proof is inspired by [CP21, §9.4] and by [KP17, Theorem 7.1.1].

Proof. We let $V \subseteq U$ be the open subsets of $T$ given by Lemma 2.3.6 and Lemma 2.3.7. By the maximal variation assumption, shrinking $V$ if necessary, we may assume that for any $t \in V$, there are only finitely many $t^{\prime} \in V$ such that $\left(X_{t}, D_{t}\right) \cong\left(X_{t^{\prime}}, D_{t^{\prime}}\right)$.

If $r$ is a fixed integer divisible by the Cartier index of $-K_{X / T}-D+2 \alpha f^{*} \lambda_{f, D}$, for an arbitrary $d \in \mathbb{Z}$ we write

$$
\mathcal{M}_{d}:=\mathcal{O}_{X}\left(d r\left(-K_{X / T}-D+2 \alpha f^{*} \lambda_{f, D}\right)\right), \quad \mathcal{M}_{d}^{D^{i}}:=\left.\mathcal{M}_{d}\right|_{D^{i}}
$$

We choose an integer $r \geq 2$ such that for every $d>0$ :

(a) $-d r\left(K_{X / T}+D\right)$ and $d r \alpha \cdot \lambda_{f, D}$ are Cartier;

(b) $\mathcal{M}_{d}$ is $f$-very ample;

(c) $R^{j} f_{*} \mathcal{M}_{d}=0$ for all $j \geq 1$;

(d) for each $i \geq 1:\left.\left(R^{j}\left(f_{i}\right)_{*} \mathcal{M}_{d}^{D^{i}}\right)\right|_{V}=0$ for all $j \geq 1$;

(e) for each $i \geq 1: f_{*} \mathcal{M}_{1} \rightarrow\left(f_{i}\right)_{*} \mathcal{M}_{1}^{D^{i}}$ is surjective on the open set $V$.

These conditions imply that $f_{*} \mathcal{M}_{1}$ and $\left.\left(\left(f_{i}\right)_{*} \mathcal{M}_{1}^{D^{i}}\right)\right|_{V}$ are locally free and compatible with base-change. In particular,

(e) if $s:=\operatorname{rk} f_{*} \mathcal{M}_{1}$, then $s=h^{0}\left(X_{t},\left.\mathcal{M}_{1}\right|_{X_{t}}\right)$ for all $t \in T$.

We may also assume that:

(f) the multiplication maps

$$
\operatorname{Sym}^{d} f_{*} \mathcal{M}_{1} \rightarrow f_{*} \mathcal{M}_{d} \quad \text { and }\left.\left.\quad \operatorname{Sym}^{d}\left(f_{*} \mathcal{M}_{1}\right)\right|_{V} \rightarrow\left(\left(f_{i}\right)_{*} \mathcal{M}_{d}^{D_{i}}\right)\right|_{V}
$$

are surjective.

Now that $r$ is chosen, we can find $d>0$ such that:

(g) For all $t \in T$, the kernel

$$
K_{t}:=\operatorname{ker}\left[\operatorname{Sym}^{d} H^{0}\left(\left.\mathcal{M}_{1}\right|_{X_{t}}\right) \longrightarrow H^{0}\left(\left.\mathcal{M}_{d}\right|_{X_{t}}\right)\right]
$$

generates $\mathcal{I}_{t}(d)$, where $\mathcal{I}_{t}$ is the ideal sheaf of $X_{t}$ for the embedding

$$
\varphi_{\left.\mathcal{M}_{1}\right|_{X_{t}}}: X_{t} \hookrightarrow \mathbb{P}^{s-1} \text {. }
$$

Here $\varphi_{\left.\mathcal{M}_{1}\right|_{X_{t}}}$ is only defined up to the action of $\operatorname{GL}_{k}(s)$ on the target. Hence, writing $w:=\operatorname{rkSym}^{d} f_{*} \mathcal{M}_{1}$ and $q_{0}:=\operatorname{rk} f_{*} \mathcal{M}_{d}$, we see that the orbit of $K_{t}$ in $\operatorname{Gr}_{k}\left(w, q_{0}\right) / \mathrm{GL}_{k}(s)$ determines the projective embedding $\varphi_{\left.\mathcal{M}_{1}\right|_{X_{t}}}$ of $X_{t}$ up to linear automorphisms of $\mathbb{P}^{s-1}$.

(h) Similarly, for all $v \in V$ and $i \geq 1$, the kernel

$$
K_{v}^{D^{i}}:=\operatorname{ker}\left[\operatorname{Sym}^{d} H^{0}\left(\left.\mathcal{M}_{1}\right|_{X_{v}}\right) \longrightarrow H^{0}\left(\left.\mathcal{M}_{d}^{D^{i}}\right|_{\left(D^{i}\right)_{v}}\right)\right]
$$

generates $\mathcal{I}_{v, i}(d)$, where $\mathcal{I}_{v, i}$ is the ideal sheaf of $\left(D^{i}\right)_{v}$ for the embedding

$$
\varphi_{\left.\mathcal{M}_{1}^{D^{i}}\right|_{\left(D^{i}\right)_{v}}}:\left(D^{i}\right)_{v} \hookrightarrow X_{v} \stackrel{\varphi_{\mathcal{M}_{1} X_{v}}}{\longrightarrow} \mathbb{P}^{s-1},
$$


Here $\varphi_{\left.\mathcal{M}_{1}^{D_{i}}\right|_{\left(D_{i}\right)_{v}}}$ is only defined up to the action of $\mathrm{GL}_{k}(s)$ on the target. Hence, writing $q_{i}:=\operatorname{rk}\left(f_{i}\right)_{*} \mathcal{M}_{d}^{D^{i}}$, we see that the orbit of $K_{v}^{D^{i}}$ in $\operatorname{Gr}_{k}\left(w, q_{i}\right) / \mathrm{GL}_{k}(s)$ determines the projective embedding of $\left(D^{i}\right)_{v}$ up to linear automorphisms of $\mathbb{P}^{s-1}$.

Having choosen $r$ and $d$ with these properties, we let

$$
W:=\left.\operatorname{Sym}^{d}\left(f_{*} \mathcal{M}_{1}\right)\right|_{U}, \quad Q_{0}:=\left.\left(f_{*} \mathcal{M}_{d}\right)\right|_{U}, \quad Q_{i}:=\left.\left(\left(f_{i}\right)_{*} \mathcal{M}_{d}^{D^{i}}\right)\right|_{U}(i \geq 1) .
$$

The sheaves $W$ and $Q_{0}$ are locally free over $U$ by construction. Since $D^{i}$ is reduced the invertible sheaf $\mathcal{M}_{d}^{D^{i}}$ satisfies the Serre condition $S_{1}$, thus its sections are supported on entire components, and each component of $D^{i}$ dominates $T$ by Lemma 2.3.3. It follows that for $i \geq 1$, the $\mathcal{O}_{T}$-modules $\left(f_{i}\right)_{*} \mathcal{M}_{d}^{D^{i}}$ are torsion-free. Since $T$ is normal, the $\left(f_{i}\right)_{*} \mathcal{M}_{d}^{D^{i}}$ are therefore locally free at codimension one points of $T$. Since they are also locally free over $V$, we may restrict $U$, but keeping it a big open set, so that the $Q_{i}$ become locally free for all $i \geq 0$.

By construction there are morphisms $W \rightarrow Q_{i}$ for $i \geq 0$, defined over $U$, which are surjective over $V$. We claim that the corresponding classifying map

$$
\xi: U(k) \longrightarrow\left(\prod_{i \geq 0} \operatorname{Gr}_{k}\left(w, q_{i}\right)\right) / \mathrm{GL}_{k}(s) \quad\left(\text { here } \mathrm{GL}_{k}(s)\right. \text { acts diagonally) }
$$

is finite-to-one over $V(k)$. Fix $t \in V(k)$. The discussion in points $(g)$ and $(h)$ above shows the following. For $t^{\prime} \in V(k)$, the equality $\xi(t)=\xi\left(t^{\prime}\right)$ holds if and only if: $X_{t}$ and $X_{t^{\prime}}$ have isomorphic embeddings into $\mathbb{P}^{s-1}$, and under this isomorphism $\left(D^{i}\right)_{t}$ is sent to $\left(D^{i}\right)_{t^{\prime}}$ for every $i \geq 1$. As explained at the beginning of the proof, there are only finitely many such $t^{\prime}$. Thus $\xi$ is finite-to-one on $V(k)$. Hence by Theorem 3.0.1, given an ample line bundle $B$ on $T$, there is a non-zero morphism

$$
\left.\operatorname{Sym}^{m q}\left(\bigoplus_{j=1}^{l} \operatorname{Sym}^{d}\left(f_{*} \mathcal{M}_{1}\right)\right)\right|_{U} \longrightarrow \mathcal{O}_{U}\left(-B_{U}\right) \otimes\left(\left.\bigotimes_{i \geq 0}\left(\operatorname{det}\left(f_{i}\right)_{*} \mathcal{M}_{d}^{D^{i}}\right)\right|_{U}\right.
$$

for some integers $l, m>0$ (the precise value of $l$ is given in Theorem 3.0.1, but it is not important for our purpose). Since $U$ is a big dense open subset of $T$, and since both sides are restrictions of locally free sheaves, by reflexivity this map extends to a non-zero morphism

$$
\operatorname{Sym}^{m q}\left(\bigoplus_{j=1}^{l} \operatorname{Sym}^{d}\left(f_{*} \mathcal{M}_{1}\right)\right) \longrightarrow \mathcal{O}_{T}(-B) \otimes\left(\bigotimes_{i \geq 0}\left(\operatorname{det}\left(f_{i}\right)_{*} \mathcal{M}_{d}^{D^{i}}\right)^{\otimes m}\right)
$$

As the right-hand side is a line bundle, this map is generically surjective.

Now let $\iota: C \rightarrow T$ be a general smooth curve. We use the notations of Notation 6.1.2. As $f_{*} \mathcal{M}_{1}$ is compatible with base-change (see the beginning of the proof), we obtain

$$
\iota^{*} f_{*} \mathcal{M}_{1} \cong h_{*} \mathcal{O}_{Z}\left(\sigma^{*} r M\right) \cong h_{*} \mathcal{O}_{Z}\left(r\left(-K_{Z / C}-D_{Z}+2 \alpha h^{*} \lambda_{h, D_{Z}}\right)\right) .
$$

As $C$ is general, the general geometric fiber of $\left(Z, D_{Z}\right) \rightarrow C$ is uniformly K-stable. Thus by [CP21, Theorem 1.20], the divisor $-K_{Z / C}-D_{Z}+2 \alpha h^{*} \lambda_{h, D_{Z}}$ is $h$-ample and nef. Moreover, since we can write

$$
r\left(-K_{Z / C}-D_{Z}+2 \alpha h^{*} \lambda_{h, D_{Z}}\right)=K_{Z / C}+D_{Z}+\underbrace{(r+1)\left(-K_{Z / C}-D_{Z}+\alpha h^{*} \lambda_{h, D_{Z}}\right)+(r-1) \alpha h^{*} \lambda_{h, D_{Z}}}_{\text {nef and } h \text {-ample }},
$$

we may apply [CP21, Proposition 6.4] to obtain that $\iota^{*} f_{*} \mathcal{M}_{1}$ is nef. Hence the pullback of $\operatorname{Sym}^{m q}\left(\bigoplus_{j=1}^{l} \operatorname{Sym}^{d}\left(f_{*} \mathcal{M}_{1}\right)\right)$ to $C$ is also nef. By generality of $C$, the restriction of the morphism (6.2.1.d) to $C$ is generically surjective, so we obtain that

$$
\left.\left.\mathcal{O}_{T}(-B)\right|_{C} \otimes\left(\bigotimes_{i \geq 0}\left(\operatorname{det}\left(f_{i}\right)_{*} \mathcal{M}_{d}^{D^{i}}\right)^{\otimes m}\right)\right|_{C} \text { is nef for a general movable } C \rightarrow T \text {. }
$$


This shows that the line bundle $\mathcal{O}_{T}(-B) \otimes\left(\bigotimes_{i \geq 0}\left(\operatorname{det}\left(f_{i}\right)_{*} \mathcal{M}_{d}^{D^{i}}\right)^{\otimes m}\right)$ is pseudo-effective. Thus $\bigotimes_{i \geq 0} \operatorname{det}\left(f_{i}\right)_{*} \mathcal{M}_{d}^{D^{i}}$ is big, and we conclude by letting $q=r d$.

\subsection{Estimation of the derivatives}

Notation 6.3.1. In the situation of Notation 6.1.1:

(a) We fix a positive integer $q$ such that the divisor $q\left(-K_{X / T}-D+2 \alpha f^{*} \lambda_{f, D}\right)$ is Cartier, and write

$$
\mathcal{N}=\mathcal{O}_{X}\left(q\left(-K_{X / T}-D+2 \alpha f^{*} \lambda_{f, D}\right)\right) \quad \text { and } \quad \mathcal{N}^{D^{i}}=\left.\mathcal{N}\right|_{D^{i}} .
$$

(b) According to Proposition 6.2.1, we may and will choose $q$ such that

$$
\bigotimes_{i \geq 0} \operatorname{det}\left(f_{i}\right)_{*} \mathcal{N}^{D^{i}} \text { is a big line bundle. }
$$

(c) If $C \rightarrow T$ is a smooth curve and $Z=X \times_{T} C$ as in Notation 6.1.2, then we let $\mathcal{N}_{Z}:=\sigma^{*} \mathcal{N}$. Recall from (6.1.2.c) that $\mathcal{N}_{Z} \cong \mathcal{O}_{Z}\left(q\left(-K_{Z / C}-D_{Z}+2 \alpha h^{*} \lambda_{f, D_{Z}}\right)\right)$, and this line bundle is nef if $C$ meets the open locus of uniformly K-stable fibers [CP21, Theorem 1.20].

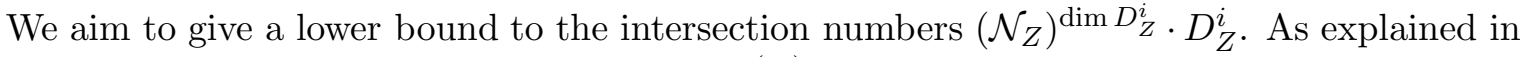
Section 1.1, the idea is to construct a product $D^{\left(r_{\bullet}\right)}$ over $T$ and then base-change over a general curve. In view of Lemma 7.0.5, we want the pullback of $D^{\left(r_{\bullet}\right)}$ to be flat over that curve. Hence it would be convenient that the restricted morphisms $D^{i} \rightarrow T$ are flat already. To achieve this, we pass to a birational model of $X$. Unfortunately this makes the notation quite cumbersome.

Notation 6.3.2. In the situation of Notation 6.3.1. Let $r_{i}$ be the generic rank of $\left(f_{i}\right)_{*} \mathcal{N}^{D^{i}}$ $(i=0, \ldots, N)$.

(a) We let

$$
D^{\left(r_{\bullet}\right)}:=\left(D^{0}\right)^{\left(r_{0}\right)} \times_{T} \cdots \times_{T}\left(D^{N}\right)^{\left(r_{N}\right)} .
$$

The projection morphism from $D^{\left(r_{\bullet}\right)}$ to the $i^{\text {th }} D^{j}$-factor is denoted by $p^{i j}: D^{\left(r_{\bullet}\right)} \rightarrow D^{j}$. We denote

$$
D_{\text {red }}^{\left(r_{\bullet}\right)}:=\left(D^{\left(r_{\bullet}\right)}\right)_{\text {red }}, \quad D_{\text {norm }}^{\left(r_{\bullet}\right)}:=\text { normalization of } D_{\text {red }}^{\left(r_{\bullet}\right)} .
$$

We denote by $g: D^{\left(r_{\bullet}\right)} \rightarrow T, g_{\text {red }}: D_{\text {red }}^{\left(r_{\bullet}\right)} \rightarrow T$ and $g_{\text {norm }}: D_{\text {norm }}^{\left(r_{\bullet}\right)} \rightarrow T$ the structural morphisms.

We define the line bundles

$$
\mathcal{N}^{\left(r_{\bullet}\right)}=\bigotimes_{i, j}\left(p^{i j}\right)^{*} \mathcal{N}^{D^{j}}
$$

and

$$
\mathcal{N}_{\text {red }}^{\left(r_{\bullet}\right)}:=\text { pullback of } \mathcal{N}^{\left(r_{\bullet}\right)} \text { to } D_{\text {red }}^{\left(r_{\bullet}\right)}, \quad \mathcal{N}_{\text {norm }}^{\left(r_{\bullet}\right)}:=\text { pullback of } \mathcal{N}^{\left(r_{\bullet}\right)} \text { to } D_{\text {norm }}^{\left(r_{\bullet}\right)} .
$$

(b) Next we fix a small $\mathbb{Q}$-factorial proper model $\mu: Y \rightarrow X$. There exists one since $X$ has a klt structure, see $[K o l 13,1.37]$. Denote by $D_{Y}=\sum_{i=1}^{N} c_{i} D_{Y}^{i}$ the strict transform of $D$. Let $\mu^{i}: D_{Y}^{i} \rightarrow D^{i}$ be the induced birational morphisms, with $\mu^{0}=\mu$. We write

$$
D_{Y}^{\left(r_{\bullet}\right)}:=\left(D_{Y}^{0}\right)^{\left(r_{0}\right)} \times_{T} \cdots \times_{T}\left(D_{Y}^{N}\right)^{\left(r_{N}\right)} .
$$

The projection morphism from $D_{Y}^{\left(r_{\bullet}\right)}$ to the $i^{\text {th }} D_{Y}^{j}$-factor is denoted by $p_{Y}^{i j}: D_{Y}^{\left(r_{\bullet}\right)} \rightarrow D_{Y}^{j}$.

We define the line bundles

$$
\mathcal{N}_{Y}^{D^{i}}:=\left(\mu^{i}\right)^{*} \mathcal{N}^{D^{i}} \text { and } \quad \mathcal{N}_{Y}^{\left(r_{\bullet}\right)}=\bigotimes_{i, j}\left(p_{Y}^{i j}\right)^{*} \mathcal{N}_{Y}^{D_{Y}^{j}}
$$


(c) If $\iota: C \rightarrow T$ is a smooth curve, we denote by $Y_{C}$, respectively $D_{Y_{C}}^{i}, D_{Y_{C}}^{\left(r_{\bullet}\right)}, \mathcal{N}_{Y_{C}}^{\left(r_{\bullet}\right)}, \mu_{C}^{i}, p_{Y_{C}}^{i j}$, the scheme-theoretic pullbacks along $\iota$ of $Y$, respectively $D_{Y}^{i}, D_{Y}^{\left(r_{\bullet}\right)}, \mathcal{N}_{Y}^{\left(r_{\bullet}\right)}, \mu^{i}, p_{Y}^{i j}$. Notice that

$$
D_{Y_{C}}^{\left(r_{\bullet}\right)}=D_{Y}^{\left(r_{\bullet}\right)} \times_{T} C=\left(D_{Y_{C}}^{0}\right)^{\left(r_{0}\right)} \times_{C} \cdots \times_{C}\left(D_{Y_{C}}^{N}\right)^{\left(r_{N}\right)}
$$

and that the projection morphisms $D_{Y_{C}}^{(r \bullet)} \rightarrow D_{Y_{C}}^{j}$ are exactly the $p_{Y_{C}}^{i j}$. Notice also that if $\mathcal{N}^{D_{Y_{C}}^{j}}$ is the pullback of $\mathcal{N}^{D_{Y}^{j}}$ along $D_{Y_{C}}^{j} \rightarrow D_{Y}^{j}$, then

$$
\mathcal{N}_{Y_{C}}^{\left(r_{\bullet}\right)} \cong \bigotimes_{i, j}\left(p_{Y_{C}}^{i j}\right)^{*} \mathcal{N}^{D_{Y_{C}}^{j}}
$$

The construction of parts $(a)$ and $(b)$ is summarized by the following diagram, where the arrow $D_{Y}^{\left(r_{\bullet}\right)} \rightarrow D_{\text {red }}^{\left(r_{\bullet}\right)}$ exists by Lemma 6.3.3(c) given below.

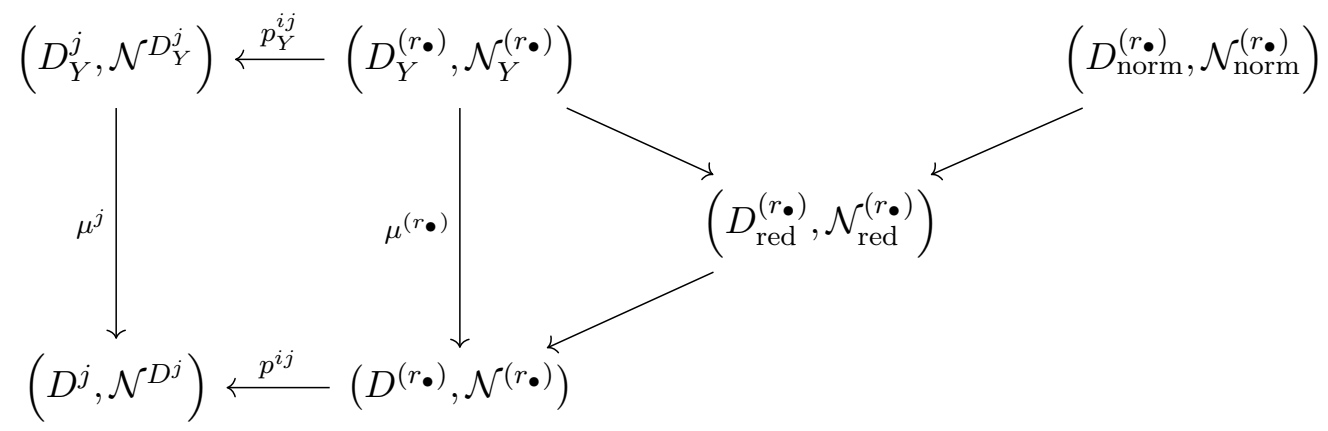

Next we establish some properties of these product varieties and these product line bundles.

Lemma 6.3.3. In the situation of Notation 6.3.2:

(a) $D^{\left(r_{\bullet}\right)}$ is equidimensional over $T$, and every component dominates $T$; moreover there is a big open set of $T$ over which $D^{\left(r_{\bullet}\right)}$ is flat and reduced;

(b) $D_{Y}^{\left(r_{\bullet}\right)}$ is reduced, flat and equidimensional over $T$, and every components dominates $T$;

(c) $\mu^{\left(r_{\bullet}\right)}: D_{Y}^{\left(r_{\bullet}\right)} \rightarrow D^{\left(r_{\bullet}\right)}$ factors through $D_{r e d}^{\left(r_{\bullet}\right)}$;

(d) $\mu^{\left(r_{\bullet}\right)}$ is an isomorphism over each generic point of $D^{\left(r_{\bullet}\right)}$, and every component of $D_{Y}^{\left(r_{\bullet}\right)}$ dominates a component of $D^{\left(r_{\bullet}\right)}$;

(e) $D_{Y_{C}}^{\left(r_{\bullet}\right)}$ is flat equidimensional over $C$, and it is reduced if $C$ is general movable.

Proof. Assertion (a) is proved in [KP17, Lemma 7.11], and assertion (c) will follow immediately from assertion (b).

The pair $(X, D)$ is klt by Lemma 5.0 .3 , so $\left(Y, D_{Y}\right)$ is klt and hence $Y$ is Cohen-Macaulay. The divisors $D_{Y}^{i}$ are $\mathbb{Q}$-Cartier because $Y$ is $\mathbb{Q}$-factorial, so each $D_{Y}^{i}$ is also Cohen-Macaulay [KM98, 5.25]. Hence all the morphisms $D_{Y}^{i} \rightarrow T$ are flat [Mat89, Theorem 23.1]. This implies that the morphism $D_{Y}^{\left(r_{\bullet}\right)} \rightarrow T$ is flat. The fibers of $D_{Y}^{\left(r_{\bullet}\right)} \rightarrow T$ have the same dimension, so the morphism is equidimensional. If one component of $D_{Y}^{\left(r_{\bullet}\right)}$ does not dominate $T$, then it belongs to the non-flat locus, which is empty. The generic fiber if reduced, so Lemma 7.0.3 implies that $D_{Y}^{(r \bullet)}$ is reduced. This proves the assertion (b), and assertion (e) is proved similarly.

To conclude, we must prove assertion (d). Let $V \subseteq X$ be the open subset over which $\mu: Y \rightarrow X$ is an isomorphism. Since $X$ is normal, $V$ is big. Thus $V_{i}:=V \cap D^{i}$ is dense in $D^{i}$ for all $i$, and $\mu^{\left(r_{\bullet}\right)}$ is an isomorphism over the open set $\mathcal{V}:=V^{\left(r_{0}\right)} \times_{T} V_{1}^{\left(r_{1}\right)} \times_{T} \cdots \times_{T} V_{N}^{\left(r_{N}\right)} \subset D^{\left(r_{\bullet}\right)}$. Assume that a generic point $\eta$ of $D^{\left(r_{\bullet}\right)}$ does not belong to $\mathcal{V}$. Then $\eta$ belongs to a product of the form $\left(D^{0}\right)^{\left(r_{0}\right)} \times_{T} \cdots \times_{T}\left(D^{N}\right)^{\left(r_{N}\right)}$ with one factor $D^{i}$ replaced by $D^{i}-V_{i}$. Such a product has dimension strictly smaller than $D^{\left(r_{\bullet}\right)}$ and so $\operatorname{dim} \overline{\{\eta\}}<\operatorname{dim} D^{\left(r_{\bullet}\right)}$, which contradicts equidimensionality. Thus $\eta \in \mathcal{V}$. Similarly, a component of $D_{Y}^{\left(r_{\bullet}\right)}$ that is contracted by $\mu^{\left(r_{\bullet}\right)}$ must belong to a product of the form $\left(D_{Y}^{0}\right)^{\left(r_{0}\right)} \times_{T} \cdots \times_{T}\left(D_{Y}^{N}\right)^{\left(r_{N}\right)}$ with one factor $D_{Y}^{i}$ replaced 
by $D_{Y}^{i}-f_{i}^{-1} V_{i}$. Such a product has dimension strictly smaller than $D_{Y}^{\left(r_{\bullet}\right)}$, which contradicts the equidimensionality of $D_{Y}^{(r \bullet)}$. So assertion (d) follows.

Lemma 6.3.4. In the situation of Notation 6.3.2,

(a) $\mathcal{N}_{\text {norm }}^{\left(r_{\bullet}\right)}$ is relatively ample over $T$ and pseudo-effective;

(b) for a general movable curve $C \rightarrow T$, the line bundle $\mathcal{N}_{Y_{C}}^{\left(r_{\bullet}\right)}$ is nef.

Proof. Let $\mathcal{U} \subset T$ be a non-empty open subset with the property that for all $t \in T$, the pair $\left(X_{t}, D_{t}\right)$ is uniformly K-stable. Let $C \rightarrow T$ be a smooth curve whose image intersects $\mathcal{U}$. Denote by $\mathcal{N}_{Z}$ the pullback of $\mathcal{N}$ on $Z=X \times_{T} C$, and $\mathcal{D}_{C}^{i}=D^{i} \times_{C} T$. Then by [CP21, Theorem 1.20], $\mathcal{N}_{Z}$ and $\mathcal{N}^{\mathcal{D}_{C}^{i}}=\left.\mathcal{N}_{Z}\right|_{\mathcal{D}_{C}^{i}}$ are nef. Thus the product line bundle

$$
\mathcal{N}_{Z}^{\left(r_{\bullet}\right)}=\bigotimes_{i, j}\left(p_{C}^{i j}\right)^{*} \mathcal{N}^{D_{C}^{i}} \quad \text { on } D^{\left(r_{\bullet}\right)} \times_{T} C
$$

is nef. By definition $\mathcal{N}_{Y_{C}}^{\left(r_{\bullet}\right)}$ is a pullback of $\mathcal{N}_{Z}^{\left(r_{\bullet}\right)}$, so it is also nef. This proves the second assertion.

By construction, the line bundle $\mathcal{N}^{\left(r_{\bullet}\right)}$ is relatively ample over $T$. Let $\tilde{C} \rightarrow D^{\left(r_{\bullet}\right)}$ be a smooth curve that meets $g^{-1} \mathcal{U}$ (recall that $g: D^{\left(r_{\bullet}\right)} \rightarrow T$ is the structural morphism). If $\tilde{C}$ is contracted by $g$, then $\mathcal{N}^{\left(r_{\bullet}\right)} \cdot \tilde{C}>0$ by relative ampleness. Otherwise, let $C \rightarrow T$ be the normalization of $g(\tilde{C})$. Then $\tilde{C} \rightarrow D^{\left(r_{\bullet}\right)}$ factors through $D^{\left(r_{\bullet}\right)} \times_{T} C$, on which the pullback of $\mathcal{N}^{\left(r_{\bullet}\right)}$ is nef. Thus $\tilde{C} \cdot \mathcal{N}^{\left(r_{\bullet}\right)} \geq 0$. This shows that $\mathcal{N}^{\left(r_{\bullet}\right)}$ is pseudo-effective. Since $\mathcal{N}_{\text {norm }}^{\left(r_{\bullet}\right)}$ is the pullback of $\mathcal{N}^{\left(r_{\bullet}\right)}$ through the finite morphism $D_{\text {norm }}^{\left(r_{\bullet}\right)} \rightarrow D^{\left(r_{\bullet}\right)}$, the first assertion follows.

We are now ready to estimate the intersection numbers $\left(\mathcal{N}_{Z}\right)^{\operatorname{dim} D_{Z}^{i}} \cdot D_{Z}^{i}\left(\right.$ where $\left.Z=X \times_{T} C\right)$. The first part of the proof is similar to the proof of [KP17, 7.1.1].

Proposition 6.3.5. In the situation of Notation 6.3.2, there are an ample Cartier divisor $A$ on some irreducible component $P$ of $D_{Y}^{(r \bullet)}$ and a rational number $e=e(X, D, q)>0$ with the following property: for every general movable curve $C \rightarrow T$, letting $A_{C}$ be the pullback of $A$ to $P \times_{T} C$, it holds that

$$
e(N+1) \cdot \operatorname{Vol}\left(A_{C}\right) \leq \sum_{j=0}^{N}\left(\mathcal{N}_{Z}\right)^{\operatorname{dim} D_{Z}^{j}} \cdot D_{Z}^{j}
$$

In particular, for every general movable curve $C \rightarrow T$,

$$
\exists j=j(C) \geq 0 \quad: \quad\left(\mathcal{N}_{Z}\right)^{\operatorname{dim} D_{Z}^{j}} \cdot D_{Z}^{j} \geq e \cdot \operatorname{Vol}\left(A_{C}\right) .
$$

Proof. By Lemma 6.3.3, the morphism $g: D^{\left(r_{\bullet}\right)} \rightarrow T$ is equidimensional and every component dominates $T$. Thus $g_{\text {red }}: D_{\text {red }}^{\left(r_{\bullet}\right)} \rightarrow T$ and $g_{\text {norm }}: D_{\text {norm }}^{\left(r_{\bullet}\right)} \rightarrow T$ are also equidimensional morphisms, and any components of $D_{\text {red }}^{\left(r_{\bullet}\right)}$ or $D_{\text {norm }}^{\left(r_{\bullet}\right)}$ dominates $T$.

By Lemma 6.3.3 and by the proof of Proposition 6.2.1, there is a big open subset $U \subseteq T$ over which $D^{\left(r_{\bullet}\right)}$ is flat and reduced, and the sheaves $\left.\left(f_{i}\right)_{*}\left(\mathcal{N}^{D^{i}}\right)\right|_{U}$ are locally free. Let $U^{0}:=g^{-1} U$. Since $U^{0}$ is reduced, it embeds as an open subset of $D_{\text {red }}^{\left(r_{\bullet}\right)}$. Therefore the open set $U^{0}$ is big in $D_{\text {red }}^{\left(r_{\bullet}\right)}$ and meets every component, and so the preimage of $U^{0}$ in $D_{\text {norm }}^{\left(r_{\bullet}\right)}$ is big and meets every component. 


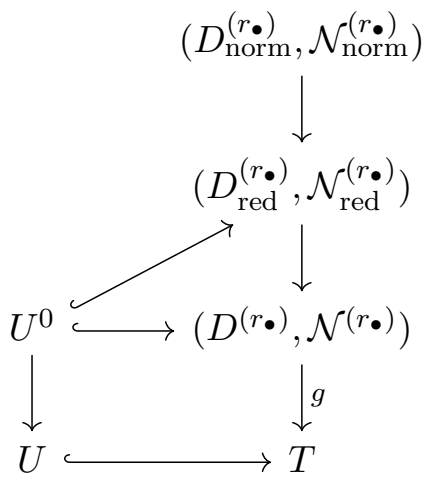

Let us write $\mathcal{L}:=\bigotimes_{i \geq 0} \operatorname{det}\left(f_{i}\right)_{*} \mathcal{N}^{D^{i}}$. On $U$, there is an embedding

$$
\left.\mathcal{L}\right|_{U} \hookrightarrow \bigotimes_{i \geq 0} \bigotimes_{j=1}^{r_{i}}\left(f_{i}\right)_{*}\left(\left.\mathcal{N}^{D^{i}}\right|_{U}\right) \cong\left(\left.g\right|_{U^{0}}\right)_{*}\left(\left.\mathcal{N}^{\left(r_{\bullet}\right)}\right|_{U^{0}}\right)
$$

where the first arrow is given by the natural embedding of det into the appropriate tensor power, and the isomorphism is given by [KP17, Lemma 3.6]. By adjunction, we obtain a morphism

$$
\left.\left(\left.g_{\text {red }}\right|_{U^{0}}\right)^{*} \mathcal{L}\right|_{U}=\left.\left.\left(\left.g\right|_{U^{0}}\right)^{*} \mathcal{L}\right|_{U} \longrightarrow \mathcal{N}^{\left(r_{\bullet}\right)}\right|_{U^{0}}=\left.\mathcal{N}_{\text {red }}^{\left(r_{\bullet}\right)}\right|_{U^{0}} .
$$

Since $U^{0}$ dominates $T$, the map (6.3.5.f) is non-zero. We may pull back this map to the normalization $D_{\text {norm }}^{\left(r_{\bullet}\right)}$. Since the preimage $U^{0}$ in $D_{\text {norm }}^{\left(r_{\bullet}\right)}$ is big, by reflexivity this pullback morphism extends to a non-zero morphism

$$
\left(g_{\text {norm }}\right)^{*} \mathcal{L} \longrightarrow \mathcal{N}_{\text {norm }}^{\left(r_{\bullet}\right)}
$$

which induces a non-zero map

$$
\mathcal{N}_{\text {norm }}^{\left(r_{\bullet}\right)} \otimes\left(g_{\text {norm }}\right)^{*} \mathcal{L} \longrightarrow\left(\mathcal{N}_{\text {norm }}^{\left(r_{\bullet}\right)}\right)^{\otimes 2} .
$$

The line bundle $\mathcal{N}_{\text {norm }}^{\left(r_{\bullet}\right)}$ is relatively ample over $T$ and pseudo-effective by Lemma 6.3.4. Moreover $\left(g_{\text {norm }}\right)^{*} \mathcal{L}$ is the pullback of a big divisor by Proposition 6.2.1. Thus the left-hand side of $(6.3 .5 . \mathrm{g})$ is big on every component by Lemma 7.0.2. Hence $\mathcal{N}_{\text {norm }}^{\left(r_{\bullet}\right)}$ is big on at least one component. This implies that $\mathcal{N}_{\text {red }}^{\left(r_{\bullet}\right)}$ is big on at least one component.

By Lemma 6.3.3, $\mu^{\left(r_{\bullet}\right)}: D_{Y}^{\left(r_{\bullet}\right)} \rightarrow D^{\left(r_{\bullet}\right)}$ is an isomorphism in codimension zero and factors through $D_{\text {red }}^{\left(r_{\bullet}\right)}$. Hence we obtain that $\mathcal{N}_{Y}^{\left(r_{\bullet}\right)}$ is big on one component $P$ of $D_{Y}^{\left(r_{\bullet}\right)}$. So we may write

$$
\left(\left.\mathcal{N}_{Y}^{\left(r_{\bullet}\right)}\right|_{P}\right)^{\otimes m} \cong \mathcal{O}_{P}(m A+m E)
$$

where $A$ is ample and $E$ is effective on $P$, for some $m>0$.

Now we fix a general movable curve $C \rightarrow T$ with the following properties. Firstly, no component of its preimage in $P$ is contained in the support of $E$. Secondly, the line bundles $\mathcal{N}_{Z}$ and $\mathcal{N}_{Y_{C}}^{\left(r_{\bullet}\right)}$ are nef (see Lemma 6.3.4). Thirdly, the induced morphisms $\mu_{C}^{j}: D_{Y_{C}}^{j} \rightarrow \mathcal{D}_{Z}^{j}:=D^{j} \times_{T}$ $C$ are birational for all $j$; this is acheviable since all $\mu^{j}: D_{Y}^{j} \rightarrow D^{j}$ are birational. Fourthly, for all $j$ the product $\mathcal{D}_{Z}^{j}$ agrees with the $j$-coefficient part $D_{Z}^{j}$ of $D_{Z}$ in codimension one; this is achievable by combining Lemma 2.3 .5 and Corollary 2.3.8. Finally, $D_{Y_{C}}^{\left(r_{\bullet}\right)}$ is equidimensional and reduced (Lemma 6.3.3.(e)).

With such a curve $C \rightarrow T$ fixed, we write

$$
\left.\mathcal{O}_{P_{C}}\left(m A_{C}+m E_{C}\right) \cong\left(\left.\mathcal{N}_{Y_{C}}^{\left(r_{\bullet}\right)}\right|_{P_{C}}\right)^{\otimes m} \cong\left(\bigotimes_{i, j}\left(p_{Y_{C}}^{i j}\right)^{*} \mathcal{N}^{D_{Y_{C}}^{j}}\right)\right|_{P_{C}} ^{\otimes m}
$$


Since $A$ is ample, its pullback $A_{C}$ is also ample. By our choice of $C$, the divisor $E_{C}$ is effective. Thus

$$
\operatorname{Vol}\left(A_{C}\right) \leq \operatorname{Vol}\left(A_{C}+E_{C}\right)=\left(\left.\mathcal{N}_{Y_{C}}^{\left(r_{\bullet}\right)}\right|_{P_{C}}\right)^{\operatorname{dim} P_{C}}
$$

It holds by equidimensionality that $\operatorname{dim} P_{C}=\operatorname{dim} D_{Y_{C}}^{\left(r_{\bullet}\right)}$. Using [Kol96, VI.2.7.3], we see that

$$
\left(\mathcal{N}_{Y_{C}}^{\left(r_{\bullet}\right)}\right)^{\operatorname{dim} D_{Y_{C}}^{\left(r_{\bullet}\right)}}=\left(\left.\mathcal{N}_{Y_{C}}^{\left(r_{\bullet}\right)}\right|_{P_{C}}\right)^{\operatorname{dim} D_{Y_{C}}^{\left(r_{\bullet}\right)}}+\sum_{P^{\prime}}\left(\left.\mathcal{N}_{Y_{C}}^{\left(r_{\bullet}\right)}\right|_{P^{\prime}}\right)^{\operatorname{dim} D_{Y_{C}}^{\left(r_{\bullet}\right)}}
$$

where $P^{\prime}$ runs through the component of $D_{Y_{C}}^{(r \bullet)}$ not contained in $P_{C}$. Since $\mathcal{N}_{Y_{C}}^{\left(r_{\bullet}\right)}$ is nef, the sum over $P^{\prime}$ is non-negative, and therefore we obtain

$$
\operatorname{Vol}\left(A_{C}\right) \leq\left(\mathcal{N}_{Y_{C}}^{\left(r_{\bullet}\right)}\right)^{\operatorname{dim} D_{Y_{C}}^{\left(r_{\bullet}\right)}}
$$

On the other hand, by Lemma 7.0.5 we have:

$$
\left(\mathcal{N}_{Y_{C}}^{\left(r_{\bullet}\right)}\right)^{\operatorname{dim} D_{Y_{C}}^{\left(r_{\bullet}\right)}}=\sum_{i=0}^{N} d_{i}\left(\mathcal{N}^{D_{Y_{C}}^{j}}\right)^{\operatorname{dim} D_{Y_{C}}^{j}} \prod_{j \neq i}\left(\mathcal{N}_{t}^{D_{Y_{C}}^{j}}\right)^{\operatorname{dim} D_{Y_{C}}^{j}-1}
$$

for some rational numbers $d_{i}=d_{i}(X, D, q)>0$ and any closed point $t \in C$. The right-hand side of (6.3.5.i) can be simplified: observe that

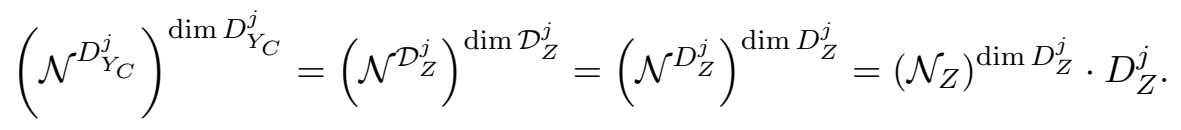

Indeed, the first equality holds because $\mu_{C}^{i}$ is birational, while the second equality holds because $\mathcal{D}_{Z}^{j}$ and $D_{Z}^{j}$ are equal in codimension 1 and $\mathcal{N}$ has full support (see [Kol96, VI.2.7.3]). Similarly to the previous displayed equalities, we also have

$$
\left(\mathcal{N}_{t}^{D_{Y_{C}}^{j}}\right)^{\operatorname{dim} D_{Y_{C}}^{j}-1}=\left(\mathcal{N}^{D_{Y_{C}}^{j}}\right)^{\operatorname{dim} D_{Y_{C}}^{j}-1} \cdot\left(D_{Y_{C}}\right)_{t}=\left(\mathcal{N}_{Z}\right)^{\operatorname{dim} D_{Z}^{j}-1} \cdot\left(D_{Z}^{j}\right)_{t}
$$

for $t \in C$ closed. Since $\mathcal{N}_{Z}$ is the pullback of a relatively ample line bundle over $T$, the $\left(\mathcal{N}_{Z}\right)^{\operatorname{dim} D_{Z}^{j}-1} \cdot\left(D_{Z}^{j}\right)_{t}$ are positive. Moreover $\mathcal{N}_{Z}$ is nef so the quantities $\left(\mathcal{N}_{Z}\right)^{\operatorname{dim} D_{Z}^{j}} \cdot D_{Z}^{j}$ are non-negative. Therefore, setting $a=\max _{j}\left\{\left(\mathcal{N}_{Z}\right)^{\operatorname{dim} D_{Z}^{j}-1} \cdot\left(D_{Z}^{j}\right)_{t}\right\}$ and $b=\max _{i}\left\{d_{i}\right\}$, it follows from (6.3.5.h) and (6.3.5.i) that

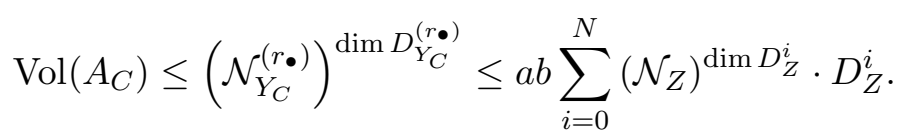

Notice that $a b$ depends only on $(X, D)$ and $q$, so we put $e=(a b(N+1))^{-1}$ to conclude.

We have now a lower bound on the derivatives $\left(\mathcal{N}_{Z}\right)^{\operatorname{dim} D_{Z}^{i}} \cdot D_{Z}^{i}$ in terms of the volume of the pullback divisor $A_{C}$. Of course, this depends on the curve $C \rightarrow T$, but it is possible to obtain some kind of uniformity. Indeed, the next lemma shows that $\operatorname{Vol}\left(A_{C}\right)$ cannot converge to zero when $[C]$ gets close to the boundary of the movable cone.

Lemma 6.3.6. Let $A$ be the ample $\mathbb{Q}$-Cartier divisor on the component $P$ of $D_{Y}^{\left(r_{\bullet}\right)}$ given by Proposition 6.3.5. Then there exists a big $\mathbb{Q}$-Cartier divisor $\Psi$ on $T$ such that for a general movable curve $C \rightarrow T$, we have $\operatorname{Vol}\left(A_{C}\right)=\Psi \cdot C$.

Proof. By Lemma 6.3.3 the scheme $P$ is reduced and the morphism $P \rightarrow T$ is equidimensional, say of relative dimension $d$. So we may apply Proposition 4.0.1 to $(P, A) \rightarrow T$. Namely, let $A^{\prime}$ be the pullback of $A$ to the normalization $P^{\prime}$ of $P$, and let $f^{\prime}: P^{\prime} \rightarrow T$ be the induced morphism. Then for a general smooth curve $C \rightarrow T$, we have $\operatorname{Vol}\left(A_{C}\right)=A_{C}^{d+1}=f_{*}^{\prime}\left(A^{\prime}\right)^{d+1} \cdot C$. By Lemma 7.0.1, $f_{*}^{\prime}\left(A^{\prime}\right)^{d+1}$ is big. We take $\Psi=f_{*}^{\prime}\left(A^{\prime}\right)^{d+1}$. 


\subsection{Variation of the boundary}

Notation 6.4.1. In this subsection, we follow Notation 6.3 .1 and let $\Psi$ be the big $\mathbb{Q}$-Cartier divisor on $T$ obtained in Lemma 6.3.6.

Given a general smooth curve $C \rightarrow T$, the inequality (6.3.5.e) gives a lower bound for some

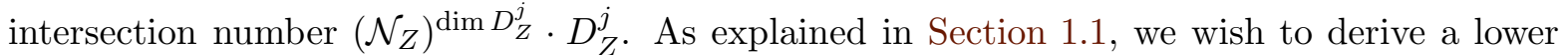
bound on $\lambda_{f, D} \cdot C$. The case $j=0$, corresponding to $D^{0}=X$, is the easiest.

Proposition 6.4.2 (Case $j=0)$. Let $C \rightarrow T$ be a smooth curve. Assume that $\left(\mathcal{N}_{Z}\right)^{n+1} \geq e \cdot(\Psi$. $C)$ for some $e>0$. Then $\lambda_{f, D} \cdot C \geq e_{0} \cdot(\Psi \cdot C)$ for some rational number $e_{0}=e_{0}(X, D, q, e)>0$.

Proof. Recall the fact that $\mathcal{N}_{Z} \cong \mathcal{O}_{Z}\left(q\left(-K_{Z / C}-D_{Z}+2 \alpha h^{*} \lambda_{f, D_{Z}}\right)\right)$ (see Notation 6.3.1). We have

$$
\begin{aligned}
\frac{1}{q^{n+1}}\left(\mathcal{N}_{Z}\right)^{n+1} & =\left(-K_{Z / C}-D_{Z}\right)^{n+1}+(n+1) 2 \alpha\left(h^{*} \lambda_{h, D_{Z}} \cdot\left(-K_{Z / C}-D_{Z}\right)^{n}\right) \\
& \left.=\operatorname{deg}_{C} h_{*}\left(\left(-K_{Z / C}-D_{Z}\right)^{n+1}\right)+(n+1) 2 \alpha \cdot \operatorname{deg} \lambda_{h, D_{Z}} \cdot\left(\left(-K_{Z / C}-D_{Z}\right)_{t}\right)^{n}\right) \\
& =\operatorname{deg}_{C} \lambda_{h, D_{Z}} \cdot[-1+2 \alpha v(n+1)] \\
& =\left(\lambda_{f, D} \cdot C\right) \cdot[\underbrace{-1+2 \alpha v(n+1)}_{>0 \text { by choice of } \alpha}]
\end{aligned}
$$

We let $e_{0}=e\left(q^{n+1}[2 \alpha v(n+1)-1]\right)^{-1}$ to obtain the desired inequality.

If $j>0$, we wish to relate $\left(\mathcal{N}_{Z}\right)^{\operatorname{dim} D_{Z}^{j}} \cdot D_{Z}^{j}$ to the a first-order derivative of $\lambda_{f, D} \cdot C$ as the component $D_{Z}^{j}$ is perturbed. Since $D_{Z}^{j}$ might not be $\mathbb{Q}$-Cartier, we introduce a birational model where it is $\mathbb{Q}$-Cartier.

Notation 6.4.3. In the situation of Notation 6.4.1. By Proposition 5.0.4, we may fix $r_{X, D} \in$ $(0 ; 1)$ with the property that for every coefficient part $D^{i}$ of $D$, there is a small birational proper morphism $W_{i} \rightarrow X$ such that for all rational numbers $\epsilon \in\left(0 ; r_{X, D}\right)$, the family $\left(W_{i}, D_{W_{i}}-\right.$ $\left.\epsilon D_{W_{i}}^{i}\right) \rightarrow T$ is a $\mathbb{Q}$-Gorenstein family of log Fano pairs of maximal variation with uniformly $\mathrm{K}$-stable general geometric fibers.

Fix an index $j>0$ and any smooth curve $\iota: C \rightarrow T$. Write $\nu: W:=W_{j} \rightarrow X$ and $\Gamma:=D^{j}$. We let

$$
V:=W \times_{X} Z \cong W \times_{T} C .
$$

Together with the notations of Notation 6.1 .2 we obtain the diagram

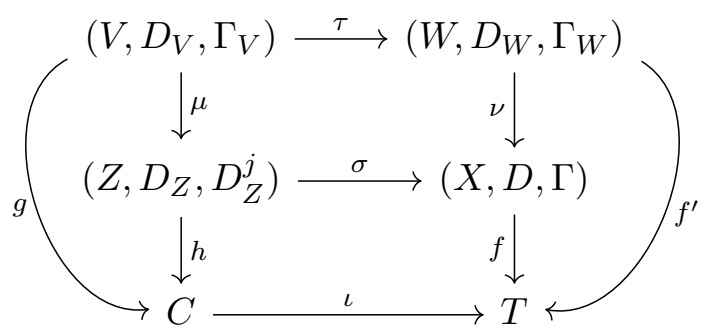

where $D_{Z}$ is the divisorial base-change of $D, D_{Z}^{j}$ the $c_{j}$-coefficient part of $D_{Z}$, and where the other $\mathbb{Q}$-divisors are defined as follows:

(a) let $D_{W}$ and $\Gamma_{W}$ be the $\nu$-strict transforms of respectively $D$ and $\Gamma$;

(b) let $D_{V}$ be defined on $V$ by the equality $K_{V / C}+D_{V}=\tau^{*}\left(K_{W / T}+D_{W}\right)$;

(c) let $\Gamma_{V}$ be defined on $V$ by $\Gamma_{V}=\tau^{*} \Gamma_{W}$. In particular, it is $\mathbb{Q}$-Cartier.

Define the polynomial $F(C, j) \in \mathbb{R}[t]$ by

$$
F(C, j)(t):=\left(-K_{V / C}-D_{V}+t \Gamma_{V}\right)^{\operatorname{dim} V}=\left(-K_{V / C}-D_{V}+t \Gamma_{V}\right)^{n+1} .
$$


We will also use the ad hoc notation

$$
\mathfrak{m}_{2}\left(\sum_{l=0}^{r} a_{l} t^{l}\right):=\max _{l \geq 2}\left\{\left|a_{l}\right|\right\} \quad \text { where } \quad \sum_{l} a_{l} t^{l} \in \mathbb{R}[t] .
$$

Lemma 6.4.4. In the situation of Notation 6.4.3. If $C \rightarrow T$ is general movable, then for every $0<\epsilon<r_{X, D}$, the family $\left(V, D_{V}-\epsilon \Gamma_{V}\right) \rightarrow C$ is a $\mathbb{Q}$-Gorenstein family of log Fanos of maximal variation with uniformly $K$-stable general general geometric fibers.

Proof. For all rational $0<\epsilon<r_{X, D}$, the family $\left(W, D_{W}-\epsilon \Gamma_{W}\right) \rightarrow T$ is a $\mathbb{Q}$-Gorenstein family of $\log$ Fanos of maximal variation with uniformly K-stable general geometric fibers. Since the fibers are normal and $C$ is normal, $V$ is also normal. If $C$ meets the open locus where the fibers are uniformly K-stable and of maximal variation, then the statement holds.

We need to relate the intersection products one can do on $V$, to the intersection products one can do on $Z$ and on $T$. This is the purpose of the next three lemmas.

Lemma 6.4.5. In the situation of Notation 6.4.3,

(a) $\mu^{*}\left(K_{Z / C}+D_{Z}\right) \sim_{\mathbb{Q}} K_{V / C}+D_{V}$.

Moreover, if $C \rightarrow T$ is a general movable curve, then

(b) $\mu: V \rightarrow Z$ is small birational;

(c) $D_{V}, \Gamma_{V}$ are the strict transforms of $D_{Z}$ and $D_{Z}^{j}$ respectively;

(d) $\Gamma_{V}$ is the divisorial pullback of $\Gamma_{W}$ and the $c_{j}$-coefficient part of $D_{V}$.

Proof. By (6.1.2.c) we have $\sigma^{*}\left(K_{X / T}+D\right) \sim_{\mathbb{Q}} K_{Z / C}+D_{Z}$, and since $\nu: W \rightarrow X$ is small we have $K_{W / T}+D_{W} \sim_{\mathbb{Q}} \nu^{*}\left(K_{X / T}+D\right)$. By definition of $D_{V}$, we obtain that $K_{V / C}+D_{V} \sim_{\mathbb{Q}}$ $\mu^{*}\left(K_{Z / C}+D_{Z}\right)$. This proves part $(a)$.

By Proposition 5.0.4, the morphism $W_{t} \rightarrow X_{t}$ is small birational for a general $t \in T$. So if $C$ meets the open locus of such $t \in T$, the morphism $\mu: V \rightarrow Z$ is birational and small as well. In this case $D_{V}, \Gamma_{V}$ are the strict transforms of $D_{Z}$ and $D_{Z}^{j}$. By Corollary 2.3.8, if $C$ is general movable then $D_{Z}^{j}$ is the divisorial pullback of $\Gamma$, and the $c_{j}$-coefficient part of $D_{Z}$. So $\Gamma_{V}$ is the $c_{j}$-coefficient part of $D_{V}$ and the divisorial pullback of $\Gamma_{W}$. This proves parts $(b),(c)$ and $(d)$.

Lemma 6.4.6. In the situation of Notation 6.4.3, if $C \rightarrow T$ is general movable, we have $\left(-K_{V / C}-D_{V}\right)^{n+1}=\left(-K_{Z / C}-D_{Z}\right)^{n+1} \quad$ and $\quad\left(-K_{V / C}-D_{V}\right)^{n} \cdot \Gamma_{V}=\left(-K_{Z / C}-D_{Z}\right)^{n} \cdot D_{Z}^{j}$.

Proof. We use Lemma 6.4.5. By part (a), it holds that $K_{V / C}+D_{V} \sim_{\mathbb{Q}} \mu^{*}\left(K_{Z / C}+D_{Z}\right)$. If $C \rightarrow T$ is general movable, $\mu$ is birational by part $(b)$. So the first equality follows. By part $(c)$, the morphism $\mu$ restricts to a birational morphism $\left.\mu\right|_{\Gamma_{V}}: \Gamma_{V} \rightarrow D_{Z}^{j}$, and the second equality follows.

Lemma 6.4.7. In the situation of Notation 6.4.3, for each $j>0$ there is a non-empty finite collection of $\mathbb{Q}$-Cartier divisors $\left\{\Upsilon_{j, l}\right\}_{l=2}^{n+1}$ on $T$ such that $\mathfrak{m}_{2}(F(C, j))=\max _{l \geq 2}\left\{\left|\Upsilon_{j, l} \cdot C\right|\right\}$ for any smooth curve $C \rightarrow T$.

Proof. Fix an index $j>0$ and let $\left(f^{\prime}: W \rightarrow T, D_{W}, \Gamma_{W}\right)$ be as in Notation 6.4.3. The fibers of $f^{\prime}$ are normal, and for small positive values of $\epsilon$, the $\mathbb{Q}$-Cartier divisor $-K_{W / T}-D_{W}+\epsilon \Gamma_{W}$ is relatively ample over $T$. Thus by Proposition 4.0.1, for any smooth curve $C \rightarrow T$ we have:

$$
f_{*}^{\prime}\left(-K_{W / T}-D_{W}+\epsilon \Gamma_{W}\right)^{n+1} \cdot C=\left(-K_{V / C}-D_{V}+\epsilon \Gamma_{V}\right)^{n+1}=F(C, j)(\epsilon) .
$$

Write $f_{*}^{\prime}\left(-K_{W / T}-D_{W}+\epsilon \Gamma_{W}\right)^{n+1}=\sum_{l=0}^{n+1} \epsilon^{l} \Upsilon_{j, l}$ in the Chow ring of $T$. By linearity of the intersection product and of $f_{*}^{\prime}$, one can describe $\Upsilon_{j, l}$ as a multiple of the pushforwards along $f^{\prime}$ of the intersection $\left(-K_{W / T}-D_{W}\right)^{n+1-l} \cdot \Gamma_{W}^{l}$. We obtain

$$
\mathfrak{m}_{2}(F(C, j))=\max _{l \geq 2}\left\{\left|\Upsilon_{j, l} \cdot C\right|\right\}
$$

as claimed. Notice that the family $\left\{\Upsilon_{j, l}\right\}_{l=2}^{n+1}$ is non-empty since $n \geq 1$. 
We are now able to treat the case $j>0$.

Proposition 6.4.8 (Case $j>0$ ). Let $C \rightarrow T$ be a general movable smooth curve. Assume that $\left(\mathcal{N}_{Z}\right)^{n} \cdot D_{Z}^{j} \geq e \cdot(\Psi \cdot C)$ for some $j>0$ and $e>0$. Then there exists a rational number $e_{1}=e_{1}(X, D, q, e)>0$ such that $\lambda_{f, D} \cdot C \geq e_{1} \cdot(\Psi \cdot C)$.

Proof. By generality $C \rightarrow T$, we may and will assume that the results of Lemma 6.4.4, Lemma 6.4.5 and Lemma 6.4.6 hold. Thus we have

$$
F(C, j)(0)=\left(-K_{Z / C}-D_{Z}\right)^{n+1}=-\operatorname{deg}_{C} \lambda_{h, D_{Z}}
$$

and

$$
F^{\prime}(C, j)(0)=(n+1)\left(-K_{Z / C}-D_{Z}\right)^{n} \cdot D_{Z}^{j}
$$

A direct calculation gives

$$
\left(\mathcal{N}_{Z}\right)^{n} \cdot D_{Z}^{j}=q^{n}\left(-K_{Z / C}-D_{Z}\right)^{n} \cdot D_{Z}^{j}+2 n \alpha \cdot \operatorname{deg}_{C} \lambda_{h, D_{Z}} \cdot\left(\mathcal{N}_{t}^{D^{j}}\right)^{n-1}
$$

Combining (6.4.8.k), (6.4.8.1) and the hypothesis on $\left(\mathcal{N}_{Z}\right)^{n} \cdot D_{Z}^{j}$, we obtain that

$$
F^{\prime}(C, j)(0) \geq \frac{(n+1) e}{q^{n}}(\Psi \cdot C)-\frac{2 n(n+1) \alpha}{q^{n}}\left(\mathcal{N}_{t}^{D^{j}}\right)^{n-1} \operatorname{deg}_{C} \lambda_{h, D_{Z}} \cdot
$$

On the other hand, for any rational $0<\epsilon<r_{X, D}$, the family $\left(V, D_{V}-\epsilon \Gamma_{V}\right) \rightarrow C$ is a $\mathbb{Q}$-Gorenstein family of log Fano pairs of maximal variation with uniformly K-stable general geometric fibers. Thus

$$
-\operatorname{deg}_{C} \lambda_{h, D_{V}-\epsilon \Gamma_{V}}=F(C, i)(\epsilon) \leq 0 \quad \forall \epsilon \in\left(0, r_{X, D}\right)
$$

by [CP21, Theorem 1.8.a]. To conclude the proof, we are going to combine (6.4.8.m) and (6.4.8.n) to get a negativity condition on $F(C, i)(0)=\operatorname{deg} \lambda_{h, D_{z}}$.

For convenience, let us write

$$
\beta_{0}=\sup \left\{\left(\mathcal{N}_{t}^{D^{i}}\right)^{n-1} \mid i>0, t \in C(k)\right\}, \quad w=\Psi . C, \quad a=\frac{(n+1) e}{q^{n}}, \quad b=\frac{2 n(n+1) \alpha}{q^{n}} \beta_{0}
$$

(notice that, by generic flatness and Noetherianity, $\beta_{0}$ is finite and actually a maximum). Therefore (6.4.8.m) implies that

$$
F^{\prime}(C, j)(0) \geq a w-b \operatorname{deg}_{C} \lambda_{h, D_{Z}}
$$

Assume that

$$
\operatorname{deg}_{C} \lambda_{h, D_{Z}} \leq \frac{a}{2 b} w
$$

Then the estimate (6.4.8.0) implies

$$
F^{\prime}(C, j)(0) \geq \frac{a}{2} w>0 .
$$

To summarize, we know by (6.4.8.n) that $F(C, j)(t)$ must be negative in a neighborhood of $t=0$, and if $F(C, j)(0)$ is small we have a positive lower bound on its first derivative. This gives an upper bound on $F(C, j)(0)$. Indeed, we apply Lemma 7.0.6 with

$$
G=\frac{a}{2} w, \quad H=\mathfrak{m}_{2}(F(C, j)), \quad l=r_{X, D}, \quad d=n+1 \geq 2,
$$

and we obtain that $F(C, j)$ takes a strictly positive value on $\left[0 ; r_{X, D} / 2\right)$ if

$$
F(C, j)(0)>\max \left\{-\frac{a r_{X, D}}{2} w,-\frac{a^{2}}{4 n} \frac{w^{2}}{\mathfrak{m}_{2}(F(C, j))}\right\},
$$

where we set $\frac{1}{\mathfrak{m}_{2}(F(C, j))}=+\infty$ if $\mathfrak{m}_{2}(F(C, j))=0$. But if (6.4.8.q) holds, then we get a contradiction with (6.4.8.n). Thus either (6.4.8.p) fails, or (6.4.8.p) holds and (6.4.8.q) fails. This can be synthetized as

$$
\operatorname{deg}_{C} \lambda_{h, D_{Z}}=-F(C, j)(0) \geq \min \left\{\frac{a}{2 b} w, \frac{a r_{X, D}}{2} w, \frac{a^{2}}{4 n} \frac{w^{2}}{\mathfrak{m}_{2}(F(C, j))}\right\} .
$$


To conclude, we need to modify the right-hand side of (6.4.8.r) so that the only quantity that depends on $C$ is $w=(\Psi . C)$. The only problematic term is $\frac{w^{2}}{\mathfrak{m}_{2}(F(C, j))}$ : it can be dealt with using Lemma 6.4.7, as we explain now.

Let $\left\{\Upsilon_{r, s}\right\}_{r, s}$ be the collection of $\mathbb{Q}$-Cartier divisors on $T$ given by Lemma 6.4.7 when considering every index $r>0$. Consider the function

$$
\overline{\operatorname{Mov}}(T)_{\mathbb{R}}-\{\mathbf{0}\} \rightarrow \mathbb{R}, \quad \gamma \mapsto \frac{\max _{r, s}\left|\Upsilon_{r, s} \cdot \gamma\right|}{\Psi \cdot \gamma} .
$$

This function is well-defined since $\Psi$ is big and hence defines a strictly positive functional on $\overline{\operatorname{Mov}}(T)_{\mathbb{R}}-\{\mathbf{0}\}$. It is also continuous and invariant under $\mathbb{R}_{+}^{*}$-scaling of its argument. So it admits a maximum which is strictly positive, since the numerator is not zero for all movable curves. Thus there exists $\beta_{1}>0$ such that

$$
\frac{\mathfrak{m}_{2}(F(C, j))}{w}=\frac{\max _{s}\left|\Upsilon_{j, s} \cdot C\right|}{\Psi \cdot C} \leq \frac{\max _{r, s}\left|\Upsilon_{r, s} \cdot C\right|}{\Psi \cdot C}<\beta_{1}
$$

for all general movable curve $C$ and $j>0$. So (6.4.8.r) implies that

$$
\operatorname{deg}_{C} \lambda_{h, D_{Z}}=-F(C, j)(0) \geq \min \left\{\frac{a}{2 b}, \frac{a r_{X, D}}{2}, \frac{a^{2}}{4 n \beta_{1}}\right\} \cdot w .
$$

The quantity $e_{1}=\min \left\{\frac{a}{2 b}, \frac{a r_{X, D}}{2}, \frac{a^{2}}{4 n \beta_{1}}\right\}$ depends only on $X, D, q$ and $e$. Therefore the proof is complete.

\subsection{Proof of Theorem 1.0.2}

Proof of point (c) of Theorem 1.0.2. Let $\tau: T^{\prime} \rightarrow T$ be a resolution of singularities. Then the induced family $f_{T^{\prime}}:\left(X_{T^{\prime}}, D_{T^{\prime}}\right) \rightarrow T^{\prime}$ is again a $\mathbb{Q}$-Gorenstein family of log Fano pairs of maximal variation with general geometric uniformly K-stable fibers. The morphism $\tau$ is birational and $\tau^{*} \lambda_{f, D}=\lambda_{f_{T^{\prime}}, D_{T^{\prime}}}$ by Proposition 2.4.1. So $\lambda_{f, D}$ is big if and only if $\lambda_{f_{T^{\prime}}, D_{T^{\prime}}}$ is big. Thus we may assume that $T$ is smooth to begin with. Let $C \rightarrow T$ be a general movable curve. By Proposition 6.3.5 and Lemma 6.3.6, the hypothesis of either Proposition 6.4.2 or Proposition 6.4.8 is fullfilled, with a constant $e$ that depends only on $X, D$ and $q$. Thus there is a constant $c=c(X, D, q)>0$ such that $\lambda_{f, D} \cdot C \geq c \cdot(\Psi \cdot C)$. As $\Psi$ is big, the result follows.

Proof of point (d) of Theorem 1.0.2. By the Nakai-Moishezon theorem it is enough to prove that for all normal varieties $V$ mapping finitely to $T$, we have $\left(\left.\lambda_{f, D}\right|_{V}\right)^{\operatorname{dim} V}>0$. Let $V^{\prime} \rightarrow V$ be a resolution of singularities. By (6.1.2.c) and since $\left(\left.\lambda_{f, D}\right|_{V}\right)^{\operatorname{dim} V}=\left(\left.\lambda_{f, D}\right|_{V^{\prime}}\right)^{\operatorname{dim} V^{\prime}}$, we may replace $f:(X, D) \rightarrow T$ by $f_{V^{\prime}}:\left(X_{V^{\prime}}, D_{V^{\prime}}\right) \rightarrow V^{\prime}$. By assumption all the closed fibers of $f_{V^{\prime}}$ are uniformly K-stable, hence klt. So all the fibers of $f_{V^{\prime}}$ are klt. Therefore we are in position to apply point (c) of Theorem 1.0.2.

\section{APPENDIX}

We gather some technical results that are used in the text.

Lemma 7.0.1. Let $f: X \rightarrow T$ be an equidimensional proper morphism of relative dimension $n$ between projective schemes. Assume that $T$ is smooth. Let $A$ be an ample $\mathbb{Q}$-Cartier divisor on $X$. Then the cycle $f_{*} A^{n+1}$ is $\mathbb{Q}$-Cartier and big. (Here $f_{*}$ denotes the cycle-theoretic pushforward.)

Proof. Since $f_{*}$ is linear, we may replace $A$ by a multiple and assume it is very ample. If $H_{1}, \ldots, H_{n+1} \in|A|$ are general elements, then $f_{*}\left(H_{1} \cap \cdots \cap H_{n+1}\right)$ is a divisor on $T$, and it is Cartier as $T$ is smooth. Since $f_{*}$ preserves rational equivalence, we have $f_{*}\left(H_{1} \cap \cdots \cap H_{n+1}\right) \in$ $\left|f_{*} A^{n+1}\right|$. It follows that this linear system is base-point free and separates points. The result now follows from [KM98, 2.60].

Lemma 7.0.2. Let $f: X \rightarrow T$ be proper morphism between normal projective $k$-schemes. Let $A$ be a pseudo-effective relatively ample $\mathbb{Q}$-Cartier divisor on $X$, and $B$ a big $\mathbb{Q}$-Cartier divisor on $T$. Then $A+f^{*} B$ is big on every component of $X$. 
Proof. We may assume that $X$ is integral. Write $B \sim_{\mathbb{Q}} C+E$ where $C$ is ample and $E$ effective. Fix an ample divisor $H$ on $X$. Choose $\epsilon^{\prime} \in \mathbb{Q}_{+}^{*}$ small enough such that $\epsilon^{\prime} A+f^{*} C$ is ample on $X$. Then choose $\epsilon \in \mathbb{Q}_{+}^{*}$ small enough such that $A+\epsilon H$ is effective, and $\epsilon^{\prime} A+f^{*} C-\left(1-\epsilon^{\prime}\right) \epsilon H$ is still ample. We write

$$
A+f^{*} B \sim_{\mathbb{Q}} f^{*} E+\left(1-\epsilon^{\prime}\right)(A+\epsilon H)+\left(\epsilon^{\prime} A+f^{*} C-\left(1-\epsilon^{\prime}\right) \epsilon H\right)
$$

so $A+f^{*} B$ is the sum of an effective and an ample $\mathbb{Q}$-divisors. By [KM98, 2.60], it is big.

Lemma 7.0.3. Let $f: X \rightarrow T$ be a flat morphism between Noetherian schemes. Assume that $T$ is integral, and that the generic fiber of $f$ is reduced. Then $X$ is reduced.

Proof. Let $x$ be an associated point of $X$. By assumption, the local morphism $\mathcal{O}_{T, f(x)} \rightarrow \mathcal{O}_{X, x}$ is flat. If $f(x)$ is not the generic point $\eta$ of $T$, then $\mathcal{O}_{T, f(x)}$ has dimension at least one, and so its maximal ideal contains a non-zero divisor. By flatness, the image of this element is also a non-zero divisor in the maximal ideal of $\mathcal{O}_{X, x}$. This contradicts the fact that $x$ is an associated point, so $f(x)=\eta$. Now $X_{\eta}$ is reduced, so $x$ cannot be an embedded associated point. Therefore $X$ is reduced.

Lemma 7.0.4. Let $X_{i}$ be proper schemes of dimensions $n_{i}(i=1, \ldots, r)$. Set $\mathcal{X}:=X_{1} \times_{k} \cdots \times_{k}$ $X_{r}$, with projections $p_{i}$ onto its factors. There is a positive rational number $c=c\left(n_{1}, \ldots, n_{r}\right)$ with the following property: if $L_{i}$ are Cartier divisors on $X_{i}$ and $L:=\sum_{i=1}^{r} p_{i}^{*} L_{i}$, then

$$
L^{\operatorname{dim} \mathcal{X}}=c \prod_{i=1}^{r} L_{i}^{n_{i}}
$$

Proof. By induction on $r$, it suffices to consider the case $r=2$. In this case we have

$$
L^{n_{1}+n_{2}}=\left(\begin{array}{c}
n_{1}+n_{2} \\
n_{1}
\end{array}\right) \cdot\left(p_{1}^{*} L_{1}\right)^{n_{1}} \cdot\left(p_{2}^{*} L_{2}\right)^{n_{2}}=\left(\begin{array}{c}
n_{1}+n_{2} \\
n_{1}
\end{array}\right) \cdot L_{1}^{n_{1}} \cdot L_{2}^{n_{2}},
$$

as claimed. In the general case, the precise form of the constant is $c=\prod_{i=1}^{r}\left(\begin{array}{c}\sum_{k>i} n_{k} \\ n_{i}\end{array}\right)$.

Lemma 7.0.5. Let $X_{i} \rightarrow T$ be flat morphisms from proper schemes of dimension $1+n_{i}$ to a common smooth curve $(i=1, \ldots, r)$. Set $\mathcal{X}:=X_{1} \times_{T} \cdots \times_{T} X_{r}$ with projections $p_{i}$ onto its factors. Then there are positive rational numbers $d_{i}=d\left(n_{1}, \ldots, n_{r}\right)$ with the following property: if $L_{i}$ are Cartier divisors on $X_{i}$ and $L:=\sum_{i=1}^{r} p_{i}^{*} L_{i}$, then

$$
L^{\operatorname{dim} \mathcal{X}}=\sum_{i=1}^{r} d_{i} L_{i}^{n_{i}+1} \prod_{j \neq i}\left(L_{j}\right)_{t}^{n_{j}}
$$

for any closed $t \in T$.

Proof. Notice that $\operatorname{dim} \mathcal{X}=1+\sum_{j} n_{j}$. Hence $L^{\operatorname{dim} \mathcal{X}}$ is a weighted sum of $\left(p_{1}^{*} L_{1}\right)^{i_{1}} \cdots\left(p_{r}^{*} L_{r}\right)^{i_{r}}$ with $\sum_{j} i_{j}=1+\sum_{j} n_{j}$. Such a term is zero as soon as $i_{j}>1+n_{j}$ for some $j$. On the other hand, by the pigeon-hole principle, at least one $i_{j}$ is greater or equal to $1+n_{j}$. Thus:

$$
\begin{aligned}
L^{1+\sum_{j} n_{j}} & =\sum_{i=1}^{r}\left(\begin{array}{c}
1+\sum_{j} n_{j} \\
1+n_{i}
\end{array}\right)\left(p_{i}^{*} L_{i}\right)^{1+n_{i}} \cdot\left(\sum_{j \neq i} p_{j}^{*} L_{j}\right)^{\sum_{j \neq i} n_{j}} \\
& =\sum_{i=1}^{r}\left(\begin{array}{c}
1+\sum_{j} n_{j} \\
1+n_{i}
\end{array}\right) L_{i}^{1+n_{i}} \cdot\left(\left.\sum_{j \neq i} p_{j}^{*} L_{j}\right|_{p_{i}^{-1}\left(x_{i}\right)}\right)^{\sum_{j \neq i} n_{j}}
\end{aligned}
$$

where the second equality holds for any $x_{i} \in X_{i}$ by flatness of $p_{i}$. Notice that the fiber of $p_{i}: \mathcal{X} \rightarrow X_{i}$ above $x_{i}$ is naturally isomorphic to the fiber product $\times_{j \neq i}\left(X_{j}\right)_{t}$ taken over Spec $k$, where $t_{i}$ is the image of $x_{i}$ through $X_{i} \rightarrow T$. By flatness of $X_{i} \rightarrow T$, the intersection number $\left(L_{i}\right)_{t_{i}}^{n_{i}}$ does not depend on $t_{i}$. Applying Lemma 7.0.4, we get

$$
L^{\operatorname{dim} \mathcal{X}}=\sum_{i=1}^{r}\left(\begin{array}{c}
1+\sum_{j} n_{j} \\
1+n_{i}
\end{array}\right) L_{i}^{1+n_{i}} c\left(n_{1}, \ldots, \widehat{n_{i}}, \ldots, n_{r}\right) \prod_{j \neq i}\left(L_{j}\right)_{t}^{n_{j}}
$$


where $t \in T$ is any closed point. Put $d_{i}\left(n_{1}, \ldots, n_{r}\right):=\left(\begin{array}{c}1+\sum_{j} n_{j} \\ 1+n_{i}\end{array}\right) c\left(n_{1}, \ldots, \widehat{n_{i}}, \ldots, n_{r}\right)$ to conclude.

Lemma 7.0.6. Let $G>0, H \geq 0$ and $l \in(0 ; 1)$ be positive real numbers, and $d \geq 2$ be an integer. Then for every choice of real numbers $a_{0}, \ldots, a_{d}$ satisfying

$$
\max \left\{-\frac{G l}{4},-\frac{G^{2}}{4 H(d-1)}\right\}<a_{0} \leq 0, \quad a_{1} \geq G, \quad \text { and } \quad\left|a_{i}\right| \leq H \forall i \geq 2,
$$

the polynomial $p(t)=\sum_{i=0}^{d} a_{i} t^{i}$ takes a strictly positive value in the interval $(0 ; l / 2)$. (If $H=0$ we set $\left.\frac{G^{2}}{4 H(d-1)}=+\infty\right)$.

Proof. Let $a_{0}, \ldots, a_{d}$ be real numbers satisfying the prescribed conditions. We have, for $0<$ $t<1$ :

$$
\begin{aligned}
p(t) & =a_{0}+a_{1} t+\sum_{i \geq 2} a_{i} t^{i} \\
& \geq a_{0}+G t-H(d-1) t^{2} .
\end{aligned}
$$

So it it enough to prove that $q(t):=a_{0}+G t-H^{\prime} t^{2}$ takes a strictly positive value on $(0, l / 2)$, with $H^{\prime}:=H(d-1)$. First consider the special case where $H=0$. Then $a_{0}>-G l / 4$, so $q(l / 3)>G l / 12>0$. From now we assume that $H>0$. We have to show that $q(t)$ has a real positive root $t_{0} \in(0, l / 2)$ such that $q^{\prime}\left(t_{0}\right)>0$. Real roots exist if

$$
a_{0}>\frac{-G^{2}}{4 H^{\prime}} \text {. }
$$

which holds by assumption on $a_{0}$. Then the smallest positive root of $q(t)$ is

$$
t_{0}=\frac{G-\sqrt{G^{2}+4 a_{0} H^{\prime}}}{2 H^{\prime}} \text {. }
$$

Note that

$$
q^{\prime}\left(t_{0}\right)=G-2 H^{\prime} t_{0}=\sqrt{G^{2}+4 a_{0} H^{\prime}}>0 .
$$

Hence we just have to verify that $t_{0}<l / 2$. This condition is equivalent to

$$
G-l H^{\prime}<\sqrt{G^{2}+4 a_{0} H^{\prime}}
$$

This inequality is trivially satisfied if $G-l H^{\prime}<0$. If $G-l H^{\prime} \geq 0$, then it is equivalent to

$$
\frac{l\left(l H^{\prime}-G\right)}{4}-\frac{G l}{4}<a_{0}
$$

which holds because $l\left(l H^{\prime}-G\right) / 4<0$ and $-G l / 4<a_{0}$ by assumption. Therefore $q(t)$ takes a strictly positive value in $(0, l / 2)$, as desired.

\section{REFERENCES}

[ABHLX20] Jarod Alper, Harold Blum, Daniel Halpern-Leistner, and Chenyang Xu. Reductivity of the automorphism group of K-polystable Fano varieties. Invent. Math., 222(3):995-1032, 2020.

[AHLH19] Jarod Alper, Daniel Halpern-Leistern, and Jochen Heinloth. Existence of moduli spaces for algebraic stacks. 2019. ArXiv e-print, arXiv:1812.01128v3.

[Alp13] Jarod Alper. Good moduli spaces for Artin stacks. Ann. Inst. Fourier (Grenoble), 63(6):2349-2402, 2013.

[BCHM10] Caucher Birkar, Paolo Cascini, Christopher D. Hacon, and James McKernan. Existence of minimal models for varieties of log general type. J. Amer. Math. Soc., 23(2):405-468, 2010.

[Ber16] Robert J. Berman. K-polystability of $\mathbb{Q}$-Fano varieties admitting Kähler-Einstein metrics. Invent. Math., 203(3):973-1025, 2016.

[Bir19] Caucher Birkar. Anti-pluricanonical systems on Fano varieties. Ann. of Math. (2), 190(2):345-463, 2019.

[BJ20] Harold Blum and Mattias Jonsson. Thresholds, valuations, and K-stability. Adv. Math., 365:107062, 57, 2020.

[BL18] Harold Blum and Yuchen Liu. Openness of uniform K-stability in families of $\mathbb{Q}$-Fano varieties. 2018. ArXiv e-print, arXiv:1808.09070v2. To appear in Ann. Sci. Éc. Norm. Supér. 
[BLX19] Harold Blum, Yuchen Liu, and Chenyang Xu. Openness of K-semistability for Fano varieties. 2019. ArXiv e-print, arXiv:1907.02408v2.

[BX19] Harold Blum and Chenyang Xu. Uniqueness of K-polystable degenerations of Fano varieties. Ann. of Math. (2), 190(2):609-656, 2019.

[CP21] Giulio Codogni and Zsolt Patakfalvi. Positivity of the CM line bundle for families of K-stable klt Fano varieties. Invent. Math., 223(3):811-894, 2021.

[Deb01] Olivier Debarre. Higher-dimensional algebraic geometry. Universitext. Springer-Verlag, New York, 2001.

[Don02] S. K. Donaldson. Scalar curvature and stability of toric varieties. J. Differential Geom., 62(2):289349, 2002.

[FO18] Kento Fujita and Yuji Odaka. On the K-stability of Fano varieties and anticanonical divisors. Tohoku Math. J. (2), 70(4):511-521, 2018.

[Fuj19a] Kento Fujita. A valuative criterion for uniform K-stability of $\mathbb{Q}$-Fano varieties. J. Reine Angew. Math., 751:309-338, 2019.

[Fuj19b] Kento Fujita. A valuative criterion for uniform K-stability of $\mathbb{Q}$-Fano varieties. J. Reine Angew. Math., 751:309-338, 2019.

[Gro66] A. Grothendieck. Éléments de géométrie algébrique. IV. Étude locale des schémas et des morphismes de schémas. III. Inst. Hautes Études Sci. Publ. Math., (28):255, 1966.

[Har77] Robin Hartshorne. Algebraic geometry. Springer-Verlag, New York-Heidelberg, 1977. Graduate Texts in Mathematics, No. 52.

[HMX14] Christopher D. Hacon, James McKernan, and Chenyang Xu. ACC for log canonical thresholds. Ann. of Math. (2), 180(2):523-571, 2014.

[Jia20] Chen Jiang. Boundedness of $\mathbb{Q}$-Fano varieties with degrees and alpha-invariants bounded from below. Ann. Sci. Éc. Norm. Supér. (4), 53(5):1235-1248, 2020.

[KM76] Finn Faye Knudsen and David Mumford. The projectivity of the moduli space of stable curves. I. Preliminaries on "det" and "Div". Math. Scand., 39(1):19-55, 1976.

[KM98] János Kollár and Shigefumi Mori. Birational geometry of algebraic varieties, volume 134 of Cambridge Tracts in Mathematics. Cambridge University Press, Cambridge, 1998. With the collaboration of C. H. Clemens and A. Corti, Translated from the 1998 Japanese original.

[Kol90] János Kollár. Projectivity of complete moduli. J. Differential Geom., 32(1):235-268, 1990.

[Kol96] János Kollár. Rational curves on algebraic varieties, volume 32 of Ergebnisse der Mathematik und ihrer Grenzgebiete. 3. Folge. A Series of Modern Surveys in Mathematics [Results in Mathematics and Related Areas. 3rd Series. A Series of Modern Surveys in Mathematics]. Springer-Verlag, Berlin, 1996.

[Kol13] János Kollár. Singularities of the Minimal Model Program, volume 200 of Cambridge Tracts in Mathematics. 2013.

[Kol14] János Kollár. Families of varieties of general type. 2014. Available at http://http://web.math.princeton.edu/ kollar/book/modbook20170720-hyper.pdf.

[Kol19] János Kollár. Families of divisors. 2019. ArXiv e-print, arXiv:1910.00937v1.

[KP17] Sándor J. Kovács and Zsolt Patakfalvi. Projectivity of the moduli space of stable log-varieties and subadditivity of log-Kodaira dimension. J. Amer. Math. Soc., 30(4):959-1021, 2017.

[Laz04a] Robert Lazarsfeld. Positivity in algebraic geometry. I, volume 48 of Ergebnisse der Mathematik und ihrer Grenzgebiete. 3. Folge. A Series of Modern Surveys in Mathematics [Results in Mathematics and Related Areas. 3rd Series. A Series of Modern Surveys in Mathematics]. Springer-Verlag, Berlin, 2004. Classical setting: line bundles and linear series.

[Laz04b] Robert Lazarsfeld. Positivity in algebraic geometry. II, volume 49 of Ergebnisse der Mathematik und ihrer Grenzgebiete. 3. Folge. A Series of Modern Surveys in Mathematics [Results in Mathematics and Related Areas. 3rd Series. A Series of Modern Surveys in Mathematics]. Springer-Verlag, Berlin, 2004. Positivity for vector bundles, and multiplier ideals.

[Liu02] Qing Liu. Algebraic geometry and arithmetic curves, volume 6 of Oxford Graduate Texts in Mathematics. Oxford University Press, Oxford, 2002. Translated from the French by Reinie Erné, Oxford Science Publications.

[LWX18] Chi Li, Xiaowei Wang, and Chenyang Xu. Quasi-projectivity of the moduli space of smooth KählerEinstein Fano manifolds. Ann. Sci. Éc. Norm. Supér. (4), 51(3):739-772, 2018.

[LX14] Chi Li and Chenyang Xu. Special test configuration and K-stability of Fano varieties. Ann. of Math. (2), 180(1):197-232, 2014.

[LXZ21] Yuchen Liu, Chenyang Xu, and Ziquan Zhuang. Finite generation for valuations computing stability thresholds and applications to K-stability. 2021. ArXiv e-print, arXiv:2102.09405v2.

[Mat89] Hideyuki Matsumura. Commutative ring theory, volume 8 of Cambridge Studies in Advanced Mathematics. Cambridge University Press, Cambridge, second edition, 1989. Translated from the Japanese by M. Reid.

[Oda13] Yuji Odaka. The GIT stability of polarized varieties via discrepancy. Ann. of Math. (2), 177(2):645661, 2013. 
[OSS16] Yuji Odaka, Cristiano Spotti, and Song Sun. Compact moduli spaces of del Pezzo surfaces and Kähler-Einstein metrics. J. Differential Geom., 102(1):127-172, 2016.

[Pat15] Zsolt Patakfalvi. Semi-negativity of Hodge bundles associated to du bois families. J. Pure Appl. Algebra, 219:5387-5393, 2015.

[PX17] Zsolt Patakfalvi and Chenyang Xu. Ampleness of the CM line bundle on the moduli space of canonically polarized varieties. Algebr. Geom., 4(1):29-39, 2017.

[Sei50] A. Seidenberg. The hyperplane sections of normal varieties. Trans. Amer. Math. Soc., 69:357-386, 1950.

[Tia97] Gang Tian. Kähler-Einstein metrics with positive scalar curvature. Invent. Math., 130(1):1-37, 1997.

[Vie83] Eckart Viehweg. Weak positivity and the additivity of the Kodaira dimension for certain fibre spaces. In Algebraic varieties and analytic varieties (Tokyo, 1981), volume 1 of Adv. Stud. Pure Math., pages 329-353. North-Holland, Amsterdam, 1983.

[Xu20] Chenyang Xu. A minimizing valuation is quasi-monomial. Ann. of Math. (2), 191(3):1003-1030, 2020.

[XZ20] Chenyang Xu and Ziquan Zhuang. On positivity of the CM line bundle on K-moduli spaces. Ann. of Math. (2), 192(3):1005-1068, 2020.

École Polytechnique Fédérale de lausanne, SB Math CAG, MA C3 595 (BÂtiment MA), Station 8, CH-1015 Lausanne, Switzerland

Email address: quentin.posva@epfl.ch 